\title{
Forkhead box R1-mediated stress response linked to a case of human microcephaly and brain atrophy
}

Andressa Mota $^{1}$, Rui Hong ${ }^{1,2}$, Sheng-Yong Niu, Féodora L. Bertherat ${ }^{1}$, Lynne Wolfe ${ }^{3}$, Christine May Malicdan ${ }^{3}$, Thomas C. Markello ${ }^{3}$, David R. Adams ${ }^{3}$, William A. Gahl ${ }^{3}$, Christine Cheng ${ }^{1,2}$, Uwe Beffert $^{1}$ and Angela $\mathrm{Ho}^{1}$

${ }^{1}$ Department of Biology, Boston University, 24 Cummington Mall, Boston, MA 02215, USA ${ }^{2}$ Bioinformatics Program, Boston University, 24 Cummington Mall, Boston, MA 02215, USA ${ }^{3} \mathrm{NIH}$ Undiagnosed Diseases Program, Common Fund, Office of the Director, National Institutes of Health, and National Human Genome Research Institute, National Institutes of Health, Bethesda, Maryland 20892, USA.

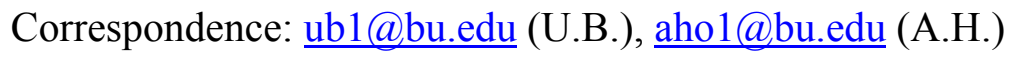

Lead Contact: aho1@bu.edu (A.H.)

Running title: FOXR1 M280L variant leads to impaired FOXR1 function 


\begin{abstract}
(175 words)
Forkhead box (Fox) family transcription factors are highly conserved and play essential roles in a wide range of cellular and developmental processes. We report an individual with severe neurological symptoms including postnatal microcephaly, progressive brain atrophy and global developmental delay associated with a de novo missense variant (M280L) in the FOXR1 gene. At the protein level, M280L impaired FOXR1 expression and induced a nuclear aggregate phenotype due to protein misfolding and proteolysis. RNAseq and pathway analysis showed that FOXR1 acts as both a transcriptional activator and repressor with central roles in heat shock response, chaperone cofactor-dependent protein refolding and cellular response to stress. Indeed, FOXR1 expression is increased in response to cellular stress, a process in which it directly controls HSPA6, HSPA1A and DHRS2 transcripts. Meanwhile, the ability of the M280L mutant to respond to stress is compromised, in part due to impaired regulation of downstream target genes that are involved in the stress response pathway. Combined, these results suggest FOXR1 plays a role in cellular stress and is necessary for normal brain development.
\end{abstract}

Keywords: aggregate/ FOXR1/ microcephaly/ stress/ transcription factor 


\section{Introduction}

Neurodevelopmental disorders result from abnormal brain development and the inability to reach cognitive, emotional and motor developmental milestones. Progress in genomics has advanced the prognosis of human neurodevelopmental disorders and provided insights into the molecular mechanisms of disease (McCarroll \& Hyman, 2013; Tarlungeanu \& Novarino, 2018; Parenti et al, 2020). While some causal genes are highly penetrant, there are also many rare single-nucleotide changes that have deleterious effects on genes of unknown function. Through exome sequencing, the NIH Undiagnosed Diseases Program (NIH UDP), a clinical site of the NIH Undiagnosed Diseases Network (UDN) identified a variant (M280L) in a single allele of the FOXR1 gene (forkhead box R1; NM_181721.2) in an individual with severe neurological symptoms including postnatal microcephaly, progressive brain atrophy and global developmental delay.

FOXR1 is a member of the evolutionarily conserved forkhead box (Fox) family of transcription factors named after the ectopic head structures observed in mutants of the Drosophila gene forkhead ( $f k h$ ) (Weigel et al, 1989; Kaestner et al, 2000; Mazet et al, 2003). Mutations in the Drosophila fkh gene cause defects in head fold involution during embryogenesis, resulting in a characteristic spiked head appearance in adult flies. Since the discovery of $f k h$, hundreds of Fox genes have been identified in organisms ranging from yeasts to humans, making it one of the largest but least explored families of higher eukaryotic transcription factors (reviewed in Hannenhalli \& Kaestner, 2009; Golson \& Kaestner, 2016). All members of the Fox gene family of transcription factors are monomeric, helix-turn-helix proteins that harbor a core fkh DNAbinding domain comprised of three $\alpha$-helices connected via a small $\beta$-sheet to a pair of loops resembling butterfly wings or a "winged-helix" (Clark et al, 1993; Gajiwala \& Burley, 2000; van Dongen et al, 2000). Despite the high degree of conservation identity in the DNA-binding domain, Fox proteins bind different target sequences with great specificity, effecting regulation of the transcription of large array of genes directing major developmental processes such as cell proliferation and cell fate specification (Clark et al., 1993; Carlsson \& Mahlapuu, 2002; Lehmann et al, 2003; Nakagawa et al, 2013). Human genetic analyses have shown several FOX genes have important biological functions associated with brain development; these include FOXG1 (potential determinant of forebrain size; Florian et al, 2012; Hettige \& Ernst, 2019; Pringsheim et al, 2019) and FOXP2 (vocal learning; MacDermot et al, 2005; Fisher \& Scharff, 2009; Nudel \& Newbury, 2013). Further, mutations in FOXG1, FOXC2, FOXL2, FOXP1 and FOXP2 have profound effects on human brain development including microcephaly, intellectual impairments, and language 
disorders (D'Haene et al, 2010; Kortum et al, 2011; Butler et al, 2012; Seltzer \& Paciorkowski, 2014; Han et al, 2019).

FOXR1, also known as FOXN5 (forkhead box N5) or DLNB13, is a 292 amino acid protein that contains an fkh DNA-binding domain (Katoh and Katoh, 2004a). Human FOXR1 and rat foxr1 gene consist of six exons with conserved exon-intron structure, indicating that FOXR1 is wellconserved between human and rat genomes (Katoh and Katoh, 2004b). The Genome-based tissue expression consortium indicate that, FOXR1 is expressed in the human brain and reproductive organs (Consortium, 2013). The Human Brain Transcriptome shows that FOXR1 is expressed in all brain regions during embryonic and postnatal development and its expression level in the brain is maintained throughout life (https://hbatlas.org). Furthermore, in situ hybridization showed that mouse foxr1 expression was present in all brain regions and enhanced within cellular nuclei, consistent with the human tissue expression profile based on the Allen Brain Atlas (Lein et al, 2007). However, little is known about the function of FOXR1. Several studies have shown that mouse foxr1 is involved in spermiogenesis (Petit et al, 2015), and several point mutations within human FOXR1 have been shown to be associated with a variety of carcinomas, although functional characterization of these oncogenic FOXR1 mutants have not been performed (Santo et al, 2012; Katoh, 2013; Pommerenke et al, 2016). Recently, foxr1 was found to be an essential maternaleffect gene in zebrafish and is required for proper cell division and survival (Cheung et al, 2018).

Here, we report a human neurodevelopmental disorder associated with a rare variant in FOXR1. We demonstrate that the de novo missense M280L variant decreases FOXR1 protein expression and displayed nuclear puncta aggregates in HEK293T cells, suggesting that impaired FOXR1 function can be pathogenic. In addition, we provide evidence that the FOXR1 M280L mutant has a compromised ability to respond to stress, in part due to impaired regulation of downstream target genes that are involved in the stress response pathway.

\section{Results}

Exome sequencing identified an individual with global developmental delay carrying a de novo missense variant in FOXR1.

The NIH UDP identified a proband with severe neurological symptoms including postnatal microcephaly, progressive brain atrophy and severe muscle hypotonia from early infancy. Brain MRI showed progressive hypoplasia in the cerebral cortex, pons and cerebellum and ventricular enlargement from age 1 to 5 (Fig 1A). The proband also exhibited growth delay, decreased body 
weight, short stature, scoliosis, hip dysplasia, ankle clonus and bell-shaped thorax (Appendix Table 1). Ophthalmic abnormalities included particular optic atrophy, cortical visual impairment and retinitis pigmentosa. Neuromuscular abnormalities included hyperactive deep tendon reflexes, joint hypermobility, severe muscle hypotonia and poor head control. The proband had myopathic facies, preauricular pits, anteverted nares and low set ears.

Exome sequencing was performed on the proband and the siblings and parents who are all unaffected. Three likely pathogenic candidate genes, rapamycin and FKBP12 target (RAFT1), ATPase $\mathrm{Na}^{+} / \mathrm{K}^{+}$transporting subunit alpha 3 (ATP1A3), and FOXR1 were identified. RAFT1 functions as a kinase that regulates cell growth, proliferation, motility, and survival (Sabatini et al, 1994; Burnett et al, 1998). The proband had a homozygous RAFT1 missense mutation, but the EXAC database identified an unaffected individual with the same RAFT1 mutation. The second candidate, ATP1A3, maintains plasma membrane sodium and potassium gradients (Heinzen et al, 2014). Investigations revealed an individual with the same mutation was discovered and a mild phenotype involving learning disability and episodes of dizziness. The last candidate is a de novo missense variant in FOXR1, a gene of unknown function, and the variant was not identified in the siblings and parents (Fig 1B). The heterozygous de novo nonsynonymous variant results in a methionine-to-leucine substitution at position 280 (M280L) and was confirmed by Sanger sequencing (Fig 1C). The M280 is found in the C-terminal segment of the FOXR1 protein, which is outside of the DNA-binding domain. M280 is highly conserved through evolution, from mammals, birds, reptiles to frogs and zebrafish (Appendix Fig 1). In addition, the M280L variant is predicted to be damaging and disease-causing based on scores of Combined Annotation Dependent Depletion (score of 29.9 where a score of 30 means that the variant is in the top $0.1 \%$ of deleterious variants in the human genome), PolyPhen-2 (score: 0.994/1.0), and Mutation Taster (score: 0.99/1.0).

\section{The FOXR1 M280L mutant leads to a decrease in FOXR1 protein expression.}

To examine whether the FOXR1 M280L mutant was properly expressed in vitro, we transiently transfected FOXR1 wild-type (WT) or the M280L mutant in HEK293T or COS7 cells and immunoblotted for FOXR1 or GFP-tagged FOXR1 protein. FOXR1 levels were significantly decreased by the M280L mutant (Fig 2A-C). Since FOXR1 is a transcription factor, we next tested whether the M280L mutant affected FOXR1 nuclear distribution in HEK293T cells transfected with either untagged or GFP-tagged FOXR1 WT or M280L. Western blot analysis demonstrated 
that both FOXR1 WT and M280L protein was expressed in both cytoplasmic and nuclear fractions with higher expression found in the nuclear fraction (Fig 2D). However, expression of the M280L mutant was reduced compared to FOXR1 WT in both cytoplasmic and nuclear fractions.

We next investigated whether the decrease in FOXR1 expression in the M280L mutant was due to transcription or protein stability changes. In HEK293T-transfected cells, we detected equal amounts of FOXR1 mRNA levels of FOXR1 WT and M280L, indicating that decreased M280L protein expression was not due to decreased transcription (Fig 2E). To measure protein stability, we blocked the proteasome pathway by treating transfected HEK293T cells with MG132, a cellpermeable proteasome inhibitor. Protein levels of both FOXR1 WT and M280L were approximately the same after proteasome inhibition, suggesting that the M280L mutant destabilizes FOXR1 protein, likely due to protein misfolding making it susceptible to proteolysis and degradation through the proteasome pathway (Fig 2F).

Finally, we investigated whether the short C-terminal tail containing M280 is necessary for protein stabilization. We produced a FOXR1 C-terminal truncation mutant lacking the last 12 amino acids from M280 ( $\Delta 280-292)$. Indeed, HEK293T cells transfected with GFP-tagged $\Delta 280-$ 292 had decreased FOXR1 protein levels, which increased following MG132 treatment, suggesting that the FOXR1 C-terminal tail is critical for FOXR1 protein stability (Fig 2G).

\section{FOXR1 M280L induces a nuclear aggregate phenotype}

To examine whether the M280L mutant alters the cellular localization of FOXR1, we transfected HEK293T cells with GFP, GFP-tagged FOXR1 WT, M280L or $\Delta 280-292$ mutant. Immunostaining for GFP showed expression of FOXR1 WT mainly in a diffuse pattern in the nucleus, co-localizing with DAPI nuclear marker (Fig 3A). In contrast, about 13\% of cells transfected with the M280L mutant formed discrete nuclear puncta (Fig 3B-C). We observed a similar phenotype in COS7 cells transfected with the M280L variant (Appendix Fig 2). In nuclei containing $>15$ puncta, the average size of individual puncta was $<2 \mu \mathrm{m}^{2}$, whereas nuclei containing $<5$ puncta had aggregates of $>4 \mu \mathrm{m}^{2}$ (Fig 3D). These results suggest that the larger puncta may form by coalescing from small nuclear foci. Expression of FOXR1 $\Delta 280-292$ mutant displayed a similar pattern, suggesting that the nuclear puncta could be due to misfolded FOXR1 protein that is susceptible to aggregation.

\section{Identification of novel FOXR1-dependent transcripts by RNA sequencing analysis}


To identify target genes regulated by FOXR1 and to investigate the role of FOXR1 M280L mutant, we performed an unbiased transcriptomic screen by RNA sequencing (RNAseq) in HEK293T cells transiently transfected with GFP, GFP-tagged FOXR1 WT or M280L. Principal component analysis showed that the three groups clustered separately excluding experimental covariates and batch effects (Appendix Fig 3A). We plotted a heat map of the log (2) fold change for all the differentially-expressed genes (DEGs) and delineated five coherent clusters (Fig 4A). Differential gene expression analysis of FOXR1 WT transfected cells compared to GFP identified 2644 DEGs of which 1315 (49.7\%) were upregulated and 1329 (50.3\%) were downregulated transcripts, suggesting that FOXR1 acts as both a transcriptional activator and repressor (Fig 4A, Appendix Fig 3B). When we compared WT to M280L, we identified 735 DEGs of which 561 (76.3\%) were upregulated and 174 (23.7\%) genes were downregulated (Appendix Fig 3B). We paid special attention to those transcripts whose levels showed a 2-fold increase in FOXR1 WT and a decrease in M280L relative to GFP control as delineated in cluster E (Fig 4B).

Gene ontology (GO) analysis for biological processes within cluster E showed genes involved in the heat shock response. These were functionally-related to negative regulation of inclusion body assembly, chaperone cofactor-dependent protein refolding, de novo protein folding, cellular response to stress and regulation of HSF1-mediated heat shock response where these were enriched in FOXR1 WT and downregulated in M280L (Fig 4C and Appendix Fig 4). Based upon the volcano plots that summarize both the expression fold-change and the statistical significance, the upregulated genes in response to FOXR1 WT and downregulated in M280L included HSPA1A and HSPA6 (both members of the Hsp70 family of heat shock proteins, Hsps), and DHRS2 (Dehydrogenase/Reductase SDR Family Member 2, a mitochondrial reductase enzyme) (Fig 4DE). These proteins play roles in protecting against oxidative stress-mediated cellular response. Quantitative real-time-PCR (qRT-PCR) supported the RNAseq data for HSPA6, HSAPA1A and DHRS2 (Fig 4F-G), confirming upregulation of gene expression in FOXR1 WT and not in the M280L mutant. Other Hsps such as SACS, DNAJC21 and DNAJC6 were increased in both FOXR1 WT and M280L groups (Fig 4G). Not all members of the Hsp70 family were misregulated in the M280L mutant; for example, HSPA12A transcript was found to be upregulated in both FOXR1 WT and the M280L mutant (Fig 4G). These results indicate that FOXR1 drives expression of specific Hsps and an important NADPH-dependent reductase enzyme that is likely related to cytoprotective pathways alleviating oxidative stress. 


\section{FOXR1 controls gene expression of heat shock chaperones and an anti-oxidant NADPH- dependent reductase}

To determine whether HSPA6, HSPA1A and DHRS2 are directly regulated by FOXR1 as opposed to secondary targets of FOXR1, we performed a de novo motif analysis of target promoters to identify consensus DNA-binding sites (Appendix Fig 5). We found strong consensus sequences for FOXR1 response elements (Nakagawa et al., 2013) within the promoter regions of at least three of the top FOXR1-regulated genes, HSPA6, HSPA1A and DHRS2 (Fig 5A). To determine whether FOXR1 expression regulates the activity of these promoters, a dual luciferase system under the control of proximal upstream regions of human HSPA6 (-1119 to -113 bp), HSPA1A (1053 to -210 bp) and DHRS2 (-3329 to -2313 bp) were co-transfected with either GFP control, FOXR1 WT or M280L mutant in HEK293T cells (Fig 5B). We found that HSPA6, HSPA1A and DHRS2 were activated by FOXR1 WT but not by M280L, indicating that these promoter regions contain FOXR1 responsive sequences and are direct targets of FOXR1 WT. The lack of luciferase activity observed in the M280L mutant is likely due to the decrease in FOXR1 expression levels (Fig 5C).

Expression of Hsps is known to be regulated by the transcription factor heat shock factor 1 (HSF1), which has a high affinity for cis-acting DNA sequence elements, including the heat shock elements (HSEs) found in the promoters of HSF-responsive genes such as Hsp70 proteins (reviewed in Akerfelt et al, 2010). There is also precedence that HSF1 target genes extend beyond molecular chaperones. For example, in C. elegans, the protective effects of reduced insulin signaling require both HSF1 and the FOXO transcription factor, DAF-16, to prevent damage by protein misfolding and to promote longevity (Hsu et al, 2003; Morley \& Morimoto, 2004; Singh \& Aballay, 2009). Based on the GO analysis for biological processes, transcripts that were upregulated in FOXR1-transfected cells were genes related to regulation of HSF1-mediated heat shock response (Appendix Fig 4). Hence, we searched and identified FOXR1 response elements upstream of the HSEs in both the HSPA6 and HSPA1A promoters (Appendix Fig 5). We also identified a consensus sequence for binding by HSF1 within the promoter region of FOXR1. Utilizing a dual luciferase system under the control of an upstream region of human FOXR1 (-633 to $+1 \mathrm{bp}$ ), FOXR1 was found to be activated by HSF1. However, HSF1-mediated FOXR1 activation was not observed when the HSF response element was mutated from TTCTAGAA to GGCTAGAA in vitro, indicating that human FOXR1 is a direct target of HSF1, which may be regulated by cellular stress (Fig 5D). 


\section{FOXR1 expression is increased in response to cellular stress}

Because FOXR1 regulates expression of HSPA6 and HSPA1A transcripts and they are also direct targets of HSF1, we hypothesized that FOXR1 expression might be directly regulated following stress-induced paradigms. We induced cellular stress using two different paradigms: serum deprivation (metabolic stress for 24 hours) and $\mathrm{CO}_{2}$-deprivation (oxidative stress for 24 hours). Under both paradigms, cells transfected with FOXR1 WT exhibited a 2.5- and 3.3-fold increase in FOXR1 protein expression under serum- and $\mathrm{CO}_{2}$-deprivation, respectively when compared to the non-stressed condition (Fig 6A). The increase in FOXR1 protein levels coincided with an increase in nuclear FOXR1 (Fig 6B). In contrast, FOXR1 M280L expression also exhibited a 3.3-fold increase under $\mathrm{CO}_{2}$-deprivation but not during serum-deprivation, indicating that the $\mathrm{M} 280 \mathrm{~L}$ mutant may be sensitive to different types of environmental stressors. In fact, the number of nuclear aggregates in cells transfected with the $\mathrm{M} 280 \mathrm{~L}$ mutant in response to $\mathrm{CO}_{2}$-deprivation was increase but not in response to serum-deprivation (Appendix movie 1).

To further explore the relationship between FOXR1 and oxidative stress, we treated FOXR1-transfected HEK293T cells with phorbol 12-myristate 13-acetate (PMA), a pharmacologic NADPH oxidase activator known to enhance reactive oxygen species (ROS) generation through a protein kinase C-mediated pathway (Bhat et al, 2019). We assessed ROS generation by fluorescence imaging using CellROX, a photostable ROS sensor. Consistent with other stress paradigms, PMA enhanced ROS generation in HEK293T cells transfected with FOXR1 WT and M280L (Figure 6C). PMA enhanced the diffuse FOXR1 expression in the nucleus of HEK293T cells transfected with FOXR1 WT. Also, the number of nuclear aggregates in cells transfected with the M280L mutant was increase by 3.88-fold compared to non-PMA treatment (two-tailed, $t=6.382 \mathrm{df}=9, \mathrm{p}<0.0001$ ), suggesting ROS-induced aggregation of FOXR1 protein in response to stress (Appendix movie 2). To determine whether ROS-induced aggregation of FOXR1 protein is cytotoxic, we measured the amount of lactate dehydrogenase (LDH) released into the medium as a measure of cytotoxicity and found no LDH changes across cells transfected with GFP alone and GFP-tagged FOXR1 WT (one-way ANOVA, $\mathrm{F}_{(3,8)}=0.2963$, p=0.6944) or FOXR1 WT compared to M280L (one-way ANOVA, $\mathrm{F}_{(3,8)}=0.2963$, $\mathrm{p}=0.8272$ ), indicating that the nuclear aggregates were not cytotoxic.

We found FOXR1 protein expression was increased 2.3- and 1.8-fold in cells transfected with FOXR1 WT and M280L mutant after PMA treatment, respectively (Fig 6D). Concomitantly, 
we found an increase in both HSPA6 and DHRS2 protein expression levels in cells transfected with FOXR1 WT (Fig 6E-F). HSPA6 levels were increased in response to PMA treatment in the M280L mutant. In contrast, we did not observe any changes in DHRS2 protein expression levels in cells transfected with M280L regardless of PMA treatment. In addition, while we observed a significant increase in HSPA1A mRNA levels in cells transfected with FOXR1 WT (Fig 4), we did not detect any changes in HSPA1A protein levels in cells transfected with FOXR1 WT or M280L. However, we did consistently see a decrease in HSPA1A protein levels in cells transfected with M280L compared to FOXR1 WT, but this difference disappeared when cells were treated with PMA.

\section{FOXR1 nuclear puncta in M280L mutant are insoluble}

To determine whether the nuclear puncta that form in HEK293T cells transfected with M280L mutant were aggresomes, which are known to serve as storage bins of misfolded or aggregated proteins (Shen et al, 2011), transfected HEK293T cells were treated with PMA and stained with the Proteostat dye. The dye detects misfolded and aggregated proteins in cells. We found bright punctate staining for proteostat-positive aggregates colocalized with the nuclear puncta in cells expressing the M280L mutant but not in FOXR1 WT (Fig 7A). These results were similar in transfected cells expressing M280L that were treated with the cell-permeable proteasome inhibitor MG132, further supporting that the M280L mutation destabilized FOXR1 protein and formed nuclear aggregates (Fig 7B).

Misfolded proteins often expose their hydrophobic domains, leading to aggregation (Kim et al, 2013; Diaz-Villanueva et al, 2015). In addition, most aggregated proteins tend to coalesce and form large deposits such as aggresomes or inclusion bodies (Kopito, 2000; Markossian \& Kurganov, 2004). Previous studies have shown that nuclear and cytoplasmic aggregates of polyQ proteins such as ataxin-1 are dynamic and exchange their components whereas ataxin-3 are immobile (Stenoien et al, 2002; Kim et al, 2016). In fact, time-lapse live cell imaging of HEK293T cells transfected with GFP-tagged M280L showed that the nuclear aggregates are quite dynamic and undergo extensive movements and fusions, with small aggregates moving toward each other and fusing to form larger aggregates (Fig 7C, Appendix movie 3).

Another criterion of misfolded proteins deposited within aggresomes is that they are largely detergent insoluble (Jensen et al, 1995; Ward et al, 1995; Scherzinger et al, 1997; Garcia-Mata et al, 1999; Kopito, 2000). Thus, we examined the biochemical properties of M280L aggregates 
versus FOXR1 WT, testing protein lysates from HEK293T cells transfected with GFP, GFPtagged FOXR1 WT or M280L for their solubility in different detergents. Protein extracts were sequentially extracted by Tris- $\mathrm{HCl}$ buffer followed by Tris- $\mathrm{HCl}$ buffer containing $1 \%$ TritonX100, then by $1 \%$ Sarkosyl and finally by $2 \%$ SDS. The amount of FOXR1 extracted in each fraction was assessed by immunoblotting for GFP-FOXR1. GFP-FOXR1 WT was detected in Tris$\mathrm{HCl}$ soluble, sarkosyl soluble and SDS soluble fractions but not present in Triton X-100, suggesting that the majority of the FOXR1 WT protein was soluble, not associated with membrane-bound proteins (Fig 7D). However, the majority of M280L was detected in the SDS fraction indicating a significant portion of the protein was insoluble and aggregating, which correlates with the increased aggregation shown by the proteostat immunolabelling.

\section{Discussion}

The UDN has identified an individual presenting with severe neurological symptoms and linked a missense variant in the FOXR1 gene as a potential variant underlying the genetic etiology of the rare neurodevelopmental disorder (Fig 1). The single de novo missense variant in FOXR1 converts a highly conserved methionine residue at amino acid 280 to leucine and was predicted to be damaging and disease-causing based on several web-based applications. Indeed, we found the M280L mutation in FOXR1 leads to a robust decrease in FOXR1 protein expression that is due to protein instability (Fig 2). Protein levels of both FOXR1 WT and M280L were approximately the same after proteasome inhibition, suggesting that the M280L mutant destabilizes FOXR1 protein, likely due to protein misfolding making it susceptible to proteolysis and degradation through the proteasome pathway. To support this finding, we found the M280L variant formed discrete nuclear puncta that colocalize with the Proteostat dye which recognizes misfolded and aggregated proteins compared to the diffuse nuclear pattern localization in FOXR1 WT (Fig 3 and 7). The M280L nuclear puncta displayed characteristic features of most aggregated proteins wherein smaller foci coalesce to form larger aggregates that were biochemically detergent-insoluble impacting on FOXR1 function (Kopito, 2000). We found the C-terminal sequences of FOXR1 are important for determining protein stability. A FOXR1 C-terminal truncation mutant lacking the last 12 amino acids from M280 ( $\triangle 280-292)$ mimics the M280L phenotype, thus suggesting that the M280L mutation most likely affected a conserved amino acid critical for protein stability.

Here we identified target genes regulated by FOXR1 based on an unbiased transcriptomic screen using RNAseq in HEK293T cells (Fig 4). Differential gene expression analysis identified 
FOXR1 acts as both a transcriptional activator and repressor. The most highly upregulated genes in response to FOXR1 WT and downregulated in M280L included two members of the Hsp70 family (HSPA1A, HSPA6) and a mitochondrial reductase enzyme, DHRS2. Each of these proteins play a role in protecting against oxidative stress-mediated cellular response. In addition, the top FOXR1-regulated genes HSPA6, HSPA1A and DHRS2 contain FOXR1 response elements within their promoter regions and are direct targets of FOXR1; M280L abolishes its ability to activate the expression of these target genes.

Cells respond to environmental stressors though the activation of specific physiological pathways that increase the abundance or activity of chaperone proteins which prevent protein misfolding to protect the proteome and maintain proteostasis (Voellmy, 1994; Grune et al, 2004; Scandalios, 2005; Raynes et al, 2016). One important mechanism is the induction of expression of Hsps such as the large Hsp70 family of proteins that help to maintain proteostasis by acting as molecular chaperones during periods of acute cellular stress (Jaattela et al, 1992; Gabai et al, 1997; Iordanskiy et al, 2004). It is well-established that HSF regulates the expression of the Hsps during times of stress where HSF binds to heat shock elements within the promoter regions of Hsps (Nollen \& Morimoto, 2002). However, there is now growing evidence that Fox family of transcription factors also influences Hsp expression. For example, the FOXO subfamily of transcription factors play an important role in protecting organisms against stress (Kops et al, 2002; Bakker et al, 2007; Eijkelenboom et al, 2013). Both FOXO genes in Drosophila (dFOXO) and in C. elegans (DAF16) are transcriptional activators for Hsp70 genes that contribute to maintaining proteostasis in response to oxidative stress. DAF16 maintains proteostasis in C. elegans by transcriptionally increasing a subset of small Hsp genes that is important in DAF-16 dependent lifespan extension (Hsu et al., 2003; Murphy et al, 2003). Consistent with C. elegans, Drosophila dFOXO also induces transcription of Hsp genes in response to oxidative stress resistance (Donovan \& Marr, 2016). Mammalian FOXO3 and FOXM1 orchestrate programs of gene expression that regulate oxidative stress resistance by upregulating catalase and MnSOD, enzymes involved in the detoxification of reactive oxygen species (Kops et al., 2002; Bakker et al., 2007; Park et al, 2009; Gurkar et al, 2018). Here, we show that FOXR1 protein expression is increased in response to metabolic and oxidative stress that increase HSPA6 and DHRS2 protein levels. We demonstrated that HSF1 bind to the FOXR1 promoter and induces its transcription, indicating FOXR1 is a direct target of HSF1. Perhaps HSF is a master transcription factor responding to stress 
and cross-talk with FOXR1 serves to fine tune transcription of target genes in response to specific stress stimuli.

An outstanding question is what role FOXR1 plays in the process of brain development. Although a preliminary diagnosis implicating the ATP1A3 variant for this patient has been made, a synergistic contribution from additional mutations including the FOXR1 M280L variant cannot be ruled out. Human genetic analyses show that several FOX transcription factors have important biological functions in brain development and mutations in FOX genes have profound effects on development and function of the brain. FOXG1, formerly named Brain Factor-1 (BF-1), is one of the earliest transcription factors expressed in the nervous cell types and tissues. FOXG1 is primarily expressed in the telencephalon and foxg1 knockout mice showed severe microcephaly with a reduction in size of the cerebral hemispheres (Xuan et al, 1995). Mechanistically, FOXG1 interacts with the global transcriptional corepressors of the Groucho/transducing-like Enhancer of the split (TLE) family suggesting that FOXG1 acts as a transcriptional repressor coordinating the control of neural progenitor cell proliferation with the timing of differentiation (Yao et al, 2000). Disruption in FOXG1 in humans leads to structural brain abnormalities including microcephaly and agenesis of the corpus callosum (Shoichet et al, 2005; Kortum et al., 2011; Hettige \& Ernst, 2019). In addition, human mutations in both FOXP1 and FOXP2 lead to severe speech and cognitive impairments (Lai et al, 2000; MacDermot et al., 2005; Takahashi et al, 2009; Horn et al, 2010; Nudel \& Newbury, 2013; Han et al., 2019); where both genes have also been linked to autism spectrum disorders (Takahashi et al., 2009; Mukamel et al, 2011; Bowers \& Konopka, 2012b,a).

The RNAseq analysis in HEK293T cells transfected with FOXR1 WT revealed some of the upregulated genes were involved in ribosome biogenesis such as ribosome biogenesis regulator 1 (RRS1) and nervous system development (MTURN, PDZD8, PTPRZ1, NOTCH2). Since HEK293T cells originate from neural crest cells, this might explain the expression of several neuron-specific genes. Ribosome biogenesis is a key driver in neurodevelopment and dysregulated ribosomal biogenesis result in neurodevelopmental syndromes that present with microcephaly, autism, intellectual deficits and/or progressive neurodegeneration (Hetman \& Slomnicki, 2019). Also, ribosome assembly is an energy-demanding process and alteration of any step in ribosomal biogenesis is highly prone to proteotoxic stress that triggers rapid activation of a specific stress pathway that coordinately upregulates heat shock target genes (Albert et al, 2019). It is possible that FOXR1 plays a role in protection against proteotoxic stress during ribosome assembly which 
is essential in brain development. We propose that FOXR1 is a transcription factor that regulates critical genes necessary during brain development which are involved in balancing growth and protein homeostasis. Therefore, understanding how FOXR1 regulates the transcription of genes and how this influences brain development are important questions to address in future experiments.

\section{Materials and Methods}

\section{Proband enrollment and consent}

The proband was evaluated at the National Institutes of Health Undiagnosed Diseases Program (NIH UDP) and was enrolled in the protocol, approved by the National Human Genome Research Institute Institutional Review Board. The parents of the proband provided written informed consent for medical and genetic studies designed to reach medical diagnoses.

\section{Exome sequencing}

Exome sequencing was performed using genomic DNA extracted from peripheral whole blood samples from the study participant and family members after informed consent onto an institutional review board approved protocol (76-HG-0238). Exome capture was carried out using manufacturer protocols using the TruSeq Exome Enrichment Kit (Illumina, San Diego, CA) and sequenced on the HiSeq 2000 Sequencing System (Illumina). Alignment to the human genome reference sequence (UCSC assembly hg19, NCBI build 37) was carried out using the Efficient Local Alignment of Nucleotide Data algorithm (Eland, Illumina, Inc) as described previously (Yuan et al, 2014). Briefly, paired-end (PE) reads were aligned independently and reads that aligned uniquely were grouped into genomic sequence intervals of $\sim 100 \mathrm{~kb}$ whereas reads that failed to align were binned with PE mates without Eland using the PE information. Reads that mapped in more than one location were discarded. To align binned reads to their respective 100 kb genomic sequence, Crossmatch, a Smith-Waterman-based local alignment algorithm was used based on the following parameters -minscore 21 and -masklevel 0 (http://www.phrap.org). Genotypes were identified using a Bayesian genotype caller, Most Probable Genotype (Teer et al, 2010). Selected de novo variants detected exclusively by exome sequencing were tested by Sanger sequencing. Sanger sequencing was performed to confirm the segregation of the identified variant 
in FOXR1 using the following primers F, 5'-AAAGCACTTCCCCTTTTTCC-3' and R, 5' AGTTGTTTGCCCATGGATTC-3’.

\section{Construction of expression vectors}

Full-length human pCMV-SPORT6 FOXR1 plasmid was purchased from GE Dharmacon (clone ID 5164198; accession \#BC038969). The human M280L mutation in FOXR1 was generated by introducing a point mutation at residue 280 (methionine to leucine) using QuikChange II SiteDirected Mutagenesis Kit (Agilent Technologies) in the pSport6 human FOXR1 plasmid with the following forward 5'-CCAACAGTGCTTGAGCCAGCCAG-3' and reverse 5'ATACTTTCTAGCCGAGTGGAAG-3' primers and verified by nucleotide sequencing. The FOXR1 wild-type and M280L mutant were then PCR amplified using forward 5'AAAGCACTCGAGATGGGGAACGAGCTCTTTCTG-3' and reverse 5'TTTGGCCCGCGGTTAAAGATCAAAGAGGAAGGG-3' primers and subcloned into the XhoI and SacII restriction sites of pEGFP-C3 (Clontech) to create an N-terminal EGFP tag. To generate the FOXR1 C-terminal truncation variant, $\Delta 280-292$, we used full-length human GFP-tagged FOXR1 wild-type as template and designed PCR forward 5'AAAGCACTCGAGATGGGGAACGAGC-3' and reverse 5'TTTGGCCCGCGGTTAGCACTGTTGGATACTTTCTAGCCG-3' primers to amplified amino acids 1-279 and subcloned into the XhoI and SacII restriction sites of pEGFP-C3.

\section{Cell culture}

HEK293T (ATCC CRL-3216) and COS-7 (ATCC CRL-1651) cells were maintained in Dulbecco's Modified Eagle's Medium(DMEM) supplemented with 10\% fetal bovine serum (FBS; Hyclone) and $1 \%$ Penicillin/Streptomycin at $37^{\circ} \mathrm{C}$ in a $5 \% \mathrm{CO}_{2}$ incubator. Cells at $60 \%$ confluency were transfected with GFP, GFP-FOXR1 or GFP-M280L plasmids using FuGENE6 transfection reagent (Promega) according to manufacturer's instructions. For subcellular fractionation, cells were briefly washed with phosphate-buffered saline (PBS) and lysed in buffer A that consists of 50 mM Tris-HCL pH 7.5, 0.5\% Triton-X100, 137.5 mM NaCl, 10\% glycerol, 5 mM EDTA pH 8.0 with proteinase inhibitors. The lysate was centrifuged $850 \times \mathrm{g}$ for $15 \mathrm{~min}$ at $4^{\circ} \mathrm{C}$. The supernatant "cytosolic" fraction was removed to a new tube and the remaining "nuclear" pellet was washed twice with buffer $\mathrm{A}$ at $4^{\circ} \mathrm{C}$ and centrifuged at $850 \mathrm{xg}$ for $2 \mathrm{~min}$. The pellet was then solubilized in buffer $B$ that consists of $50 \mathrm{mM}$ Tris-HCL pH 7.5, 0.5\% Triton-X100, $137.5 \mathrm{mM}$ 
$\mathrm{NaCl}, 10 \%$ glycerol, $5 \mathrm{mM}$ EDTA $\mathrm{pH}$ 8.0, 0.5\% SDS with proteinase inhibitors and sonicated for 5-10 secs. Equal amount of 2x sample buffer (0.1 M Tris-HCL, pH 6.8, 4\% SDS, 20\% glycerol, $10 \% \beta$-mercaptoethanol, $0.01 \%$ bromophenol blue) was added to the tubes containing the nuclear and cytoplasmic fractions, boiled at $100^{\circ} \mathrm{C}$ for $10 \mathrm{~min}$ and subjected to SDS-PAGE. For MG132 treatment, transfected cells were treated with $50 \mu \mathrm{M}$ MG132 (Sigma-Aldrich) for $24 \mathrm{~h}$. Cells were then washed with PBS and collected in 2x sample buffer. Cellular stress paradigms: serum starvation, cells were incubated in DMEM without fetal bovine serum for $24 \mathrm{~h}$ at $37^{\circ} \mathrm{C}$; for $\mathrm{CO}_{2}$ deprivation, cells were deprived of $5 \% \mathrm{CO}_{2}$ for $24 \mathrm{~h}$ at $37^{\circ} \mathrm{C}$; PMA treatment, cells were treated with $1 \mu \mathrm{M}$ of phorbol 12-myristate 13 -acetate (PMA, Sigma) for $24 \mathrm{~h}$ at $37^{\circ} \mathrm{C}$.

\section{Western blotting}

Whole cell lysates were extracted from cells in 2x sample buffer and separated on 10\% SDSPAGE gel and transferred to nitrocellulose membranes (GE Healthcare). The membranes were blocked with Odyssey Blocking Buffer in PBS (Licor), followed by incubation with primary antibodies against human FOXR1 (Biorbyt, 1:200), GFP (synaptic systems, 1:1000), GAPDH (EMD Millipore, 1:5000), HSPA1A and HSPA6 (Enzo life sciences, 1:1000), DHRS2 (Abcam, 1:500), Histone $\mathrm{H} 3$ (Cell-Signaling, 1:1000) overnight at $4^{\circ} \mathrm{C}$. Proteins recognized by the antibodies were detected with an Odyssey infrared imaging system (LI-COR) using IRDye680RDor IRDye800CW-coupled secondary antibodies (LI-COR, 1: 20,000).

\section{Immunocytochemistry and image analysis}

Transfected cells plated on coverslips were washed briefly with PBS and fixed with 4\% paraformaldehyde at room temperature for $10 \mathrm{~min}$, permeabilized and blocked in $10 \%$ goat serum, 0.1\% saponin in PBS. To detect oxidative stress following PMA treatment, transfected cells were incubated with $5 \mu \mathrm{M}$ CellROX Oxidative Stress Reagent (Thermo Fisher Scientific) for 30 min at $37^{\circ} \mathrm{C}$ prior to fixation with paraformaldehyde. To detect aggresomes in transfected cells, we used the PROTEOSTAT Aggresome Detection Kit (Enzo Life Sciences). The dye intercalates into the cross-beta spine of quaternary protein structures found in misfolded and aggregated proteins. Coverslips were mounted with ProLong Gold Anti-Fade Mount with DAPI (Fisher Scientific) and imaged with a Carl Zeiss LSM700 confocal microscope. Images were collected with identical confocal settings for all of the samples and Z-stacked images were projected with maximal projection mode using Zeiss Confocal Software. 


\section{RNA sequencing and analysis}

HEK293T were transfected with GFP, GFP-FOXR1 or GFP-M280L mutant using FuGENE6. Forty-eight hours after transfection, total RNA was purified using the QIAshredder and RNeasy Mini Kit (QIAGEN) and samples were processed with Trizol (Invitrogen). Three biological replicates were processed independently. RNA samples were suspended in DEPC-treated water and concentrations were determined using the Nanodrop ND-1000 (Thermo Scientific) where all samples showed A260/A280 ratios higher than 2.0 and RNA integrity were also checked in a bioanalyzer (Agilent 2100). Library preparations and sequencing were performed by The Broad Institute, MA using Illumina HiSeq 2000 technology.

The RNA sequencing reads were aligned to the GRCh38 Homo sapiens genome using HISAT2 (Kim et al, 2019) with default parameters. The bam files were sorted by read names instead of chromosome coordinates by SAMtools (Li et al, 2009). Gene count matrix of each sample was generated by HTSeq, a Python framework to work with high-throughput sequencing data (Anders et al, 2015). Downstream analysis was performed with the DEseq2 (Love et al, 2014) package in R. Gene that were not expressed in any cell and were removed from downstream analysis. Sample PCA plot was generated with 'plotPCA' function to detect and remove the outlier sample in each condition. Differential expression analysis between conditions was performed with the 'DESeq' function with default parameters. Log-fold change shrinkage was performed on the differential expression analysis result. DEGs with adjust-p value $<0.05$ and log-fold-change $>$ 0.25 were kept for downstream analysis. Heatmap of differentially expressed genes were visualized with heatmap and genes with similar expression patterns across samples were clustered on the heatmap. Gene set enrichment analysis was conducted with the GSEA (Subramanian et al, 2005). Gene ontology and enrichment analysis encompassing the DEGs were analyzed using the Database for Annotation, Visualization, and Integrated Discovery (DAVID v6.8) software where the threshold was set as modified Fisher Exact $P$-value (EASE score) $\leq 0.05$.

\section{Quantitative real-time qPCR analyses}

Total RNA was purified using the QIAshredder and RNeasy Mini Kit (Qiagen). The cDNA was synthesized using iScript cDNA Synthesis Kit (Biorad) or Accuris qMax cDNA Synthesis Kit (Midland Scientific). Quantitative real-time PCR was performed in an ABI Prism 7900HT Fast Real-Time PCR System (Applied Biosystems) using the Power SYBR Green PCR Master mix 
(Thermo Scientific) with a two-step cycling protocol and an annealing/extension temperature of $60^{\circ} \mathrm{C}$. The experiment was performed with three biological replicates and three technical replicates each. The relative amount for each target was normalized using GAPDH as a reference gene and the fold change in gene expression was calculated using the ddCt method with the GFP-transfected cells serving as control. Primers were as follows: DHRS2: 5'-TCATCAGCTGCAGAGGATTGG3' (forward) and 5'-AATGTTCTCCCCGTTGACGTA-3' (reverse); DNAJC6: 5' AGGACAACTTGAAAGACACCCT-3' (forward) and 5' - AAATCTCCCTTTGTGTAGCTGG3' (reverse); DNAJC21: 5'-CCTGAAATGGCACCCGGATAA-3' (forward) and 5'TTTCCTGAGGGTCACTCAACA-3' (reverse); GAPDH: 5'-GGATTTGGTCGTATTGGG-3' (forward) and 5'-GGAAGATGGTGATGGGATT-3' (reverse); HSPA1A: 5'GCCTTTCCAAGATTGCTGGTT-3' (forward) and 5'- TCAACATTGCAAACACAGGA-3' (reverse); HSPA6: 5'-CAAGGTGCGCGTATGCTAC-3' (forward) and 5'GCTCATTGATGATCCGCAACAC-3' (reverse); HSPA12A: 5'GCTCCCACATCTGCATATTCAT-3' (forward) and 5'- TTCTGAGACGTTGGAGTCAGT-3' (reverse); SACS: 5'- ACAACAACGCGGTTTTCACC-3' (forward) and 5'GCCTGATTCATGTGGGCCAA-3' (reverse). Data analysis was performed using the ABI Prism 7900HT SDS Software.

\section{Dual luciferase assay}

Promoter sequences of human HSPA1A, HSPA6, DHRS2 were amplified by PCR from genomic DNA and subcloned into pNL3.1-minP/Nluc (Promega). Cells were transfected with the normalization plasmid (pGL4.54-Luc2/TK), the reporter plasmid (pNL3.1-minP/Nluc) and GFPtagged FOXR1 or M280L plasmid. Transfected cells were collected in PBS and luciferase activity was assessed using the Nano-Glo Dual Luciferase reporter assay system (Promega). Dual luciferase signal was quantified using a VICTOR-3 plate reader (Perkin Elmer). To control for transfection efficiency, the Nluc reporter plasmid signal was normalized to the constitutive luciferase signal (i.e., signal from pGL4.54 plasmids, Nluc/Luc2). Fold-induction values for each promoter sequence were calculated relative to the background activity of reporter plasmid in the presence of GFP-FOXR1 or GFP-M280L plasmid. Reporter assays were performed as three biological replicates with three technical replicates per biological replicate.

\section{Detergent extraction assay}


Transfected HEK293T cells were isolated in $50 \mathrm{mM}$ Tris- $\mathrm{HCl}$ buffer at $\mathrm{pH} 7.5$ with protease inhibitors by brief sonication and ultracentrifuged at $350,000 \mathrm{x} g$ for $15 \mathrm{~min}$ and the supernatant was collected as a Tris-HCl soluble fraction (adapted from Kuwahara et al, 2012). The resulting pellet was sequentially extracted in Tris- $\mathrm{HCl}$ buffer containing $1 \%$ Triton $\mathrm{X}-100$, then by $1 \%$ Sarkosyl and finally by $2 \%$ SDS. Each detergent extraction step was incubated for $1 \mathrm{~h}$ at $4^{\circ} \mathrm{C}$ and ultracentrifgued at $350,000 \mathrm{x} g$ for $15 \mathrm{~min}$, resulting in a Triton X-100 soluble fraction, Sarkosyl soluble fraction and SDS soluble fraction, respectively. The Tris- $\mathrm{HCl}$ fraction containing $20 \mathrm{~g}$ of total proteins, along with equal volumes of Triton X-100, Sarkosyl and SDS fractions were loaded onto SDS-PAGE.

\section{Statistical analysis}

To determine statistical significance, we use either a Student's $t$-test to compare two groups, or a one-way repeated measures ANOVA for multiple comparisons and two-way ANOVA for comparisons with multiple variables. All bars and error bars represent the mean \pm S.E.M. and significance was set at $\mathrm{p}<0.05$. The data were analyzed using GraphPad Prism 7 software (GraphPad Software Inc., San Diego, CA).

\section{Data availability}

The accession number for the RNAseq reported in this paper is GEO:GSE

\section{Acknowledgements}

We thank the colleagues in the laboratory who have provided constructive feedback on this work. This work was supported in part by the Intramural Research Program of the National Human Genome Research Institute (HG000215 to W.A.G.) and by a National Institutes of Health grant (R21GM114629 to U.B. and A.H.).

\section{Author Contributions}

A.M., A.H., U.B. Conceptualization; A.M., R.H., S.N., C.C., L.W. Data curation; A.M., R.H., T.M. Formal analysis; A.H., U.B., W.A.G. Funding acquisition; A.M., R.H., S.N., C.M.M., C.C, U.B., A.H. Investigation; A.M., R.H., S.N., F.B., D.R.A., Methodology; D.R.A., W.A.G., C.C., U.B., A.H. Project Administration; C.M.M., D.R.A., W.A.G., C.C., U.B., A.H. Supervision; A.M., 
R.H., T.M., Validation; A.H. Writing-original draft; A.M., C.M.M., W.A.G., U.B., A.H. Writingreview \& editing

\section{Conflict of Interests}

The authors declare that they have no conflict of interest.

\section{References}

Akerfelt M, Morimoto RI, Sistonen L (2010) Heat shock factors: integrators of cell stress, development and lifespan. Nat Rev Mol Cell Biol 11: 545-555

Albert B, Kos-Braun IC, Henras AK, Dez C, Rueda MP, Zhang X, Gadal O, Kos M, Shore D (2019) A ribosome assembly stress response regulates transcription to maintain proteome homeostasis. Elife 8

Anders S, Pyl PT, Huber W (2015) HTSeq--a Python framework to work with high-throughput sequencing data. Bioinformatics 31: 166-169

Bakker WJ, Harris IS, Mak TW (2007) FOXO3a is activated in response to hypoxic stress and inhibits HIF1-induced apoptosis via regulation of CITED2. Mol Cell 28: 941-953

Bhat SA, Sood A, Shukla R, Hanif K (2019) AT2R Activation Prevents Microglia Proinflammatory Activation in a NOX-Dependent Manner: Inhibition of PKC Activation and p47(phox) Phosphorylation by PP2A. Mol Neurobiol 56: 3005-3023

Bowers JM, Konopka G (2012a) ASD-relevant Animal Models of the Foxp Family of Transcription Factors. Autism Open Access Suppl 1

Bowers JM, Konopka G (2012b) The role of the FOXP family of transcription factors in ASD. Dis Markers 33: 251-260

Burnett PE, Barrow RK, Cohen NA, Snyder SH, Sabatini DM (1998) RAFT1 phosphorylation of the translational regulators p70 S6 kinase and 4E-BP1. Proc Natl Acad Sci U S A 95: 1432-1437

Butler MG, Dagenais SL, Garcia-Perez JL, Brouillard P, Vikkula M, Strouse P, Innis JW, Glover TW (2012) Microcephaly, intellectual impairment, bilateral vesicoureteral reflux, distichiasis, and glomuvenous malformations associated with a 16q24.3 contiguous gene deletion and a Glomulin mutation. Am J Med Genet A 158A: 839-849

Carlsson P, Mahlapuu M (2002) Forkhead transcription factors: key players in development and metabolism. Dev Biol 250: 1-23 
Cheung CT, Patinote A, Guiguen Y, Bobe J (2018) foxr1 is a novel maternal-effect gene in fish that is required for early embryonic success. PeerJ 6: e5534

Clark KL, Halay ED, Lai E, Burley SK (1993) Co-crystal structure of the HNF-3/fork head DNArecognition motif resembles histone H5. Nature 364: 412-420

Consortium GT (2013) The Genotype-Tissue Expression (GTEx) project. Nat Genet 45: 580-585

D'Haene B, Nevado J, Pugeat M, Pierquin G, Lowry RB, Reardon W, Delicado A, Garcia-Minaur S, Palomares M, Courtens W et al (2010) FOXL2 copy number changes in the molecular pathogenesis of BPES: unique cohort of 17 deletions. Hum Mutat 31: E1332-1347

Diaz-Villanueva JF, Diaz-Molina R, Garcia-Gonzalez V (2015) Protein Folding and Mechanisms of Proteostasis. Int J Mol Sci 16: 17193-17230

Donovan MR, Marr MT, 2nd (2016) dFOXO Activates Large and Small Heat Shock Protein Genes in Response to Oxidative Stress to Maintain Proteostasis in Drosophila. J Biol Chem 291: 19042-19050

Eijkelenboom A, Mokry M, de Wit E, Smits LM, Polderman PE, van Triest MH, van Boxtel R, Schulze A, de Laat W, Cuppen E et al (2013) Genome-wide analysis of FOXO3 mediated transcription regulation through RNA polymerase II profiling. Mol Syst Biol 9: 638

Fisher SE, Scharff C (2009) FOXP2 as a molecular window into speech and language. Trends Genet 25: 166-177

Florian C, Bahi-Buisson N, Bienvenu T (2012) FOXG1-Related Disorders: From Clinical Description to Molecular Genetics. Mol Syndromol 2: 153-163

Gabai VL, Meriin AB, Mosser DD, Caron AW, Rits S, Shifrin VI, Sherman MY (1997) Hsp70 prevents activation of stress kinases. A novel pathway of cellular thermotolerance. J Biol Chem 272: $18033-18037$

Gajiwala KS, Burley SK (2000) Winged helix proteins. Curr Opin Struct Biol 10: 110-116

Garcia-Mata R, Bebok Z, Sorscher EJ, Sztul ES (1999) Characterization and dynamics of aggresome formation by a cytosolic GFP-chimera. J Cell Biol 146: 1239-1254

Golson ML, Kaestner KH (2016) Fox transcription factors: from development to disease. Development 143: 4558-4570

Grune T, Jung T, Merker K, Davies KJ (2004) Decreased proteolysis caused by protein aggregates, inclusion bodies, plaques, lipofuscin, ceroid, and 'aggresomes' during oxidative stress, aging, and disease. Int J Biochem Cell Biol 36: 2519-2530 
Gurkar AU, Robinson AR, Cui Y, Li X, Allani SK, Webster A, Muravia M, Fallahi M, Weissbach H, Robbins PD et al (2018) Dysregulation of DAF-16/FOXO3A-mediated stress responses accelerates oxidative DNA damage induced aging. Redox Biol 18: 191-199

Han L, Chen M, Wang Y, Wu H, Quan Y, Bai T, Li K, Duan G, Gao Y, Hu Z et al (2019) Pathogenic missense mutation pattern of forkhead box genes in neurodevelopmental disorders. Mol Genet Genomic Med 7: e00789

Hannenhalli S, Kaestner KH (2009) The evolution of Fox genes and their role in development and disease. Nat Rev Genet 10: 233-240

Heinzen EL, Arzimanoglou A, Brashear A, Clapcote SJ, Gurrieri F, Goldstein DB, Johannesson SH, Mikati MA, Neville B, Nicole S et al (2014) Distinct neurological disorders with ATP1A3 mutations. Lancet Neurol 13: 503-514

Hetman M, Slomnicki LP (2019) Ribosomal biogenesis as an emerging target of neurodevelopmental pathologies. J Neurochem 148: 325-347

Hettige NC, Ernst C (2019) FOXG1 Dose in Brain Development. Front Pediatr 7: 482

Horn D, Kapeller J, Rivera-Brugues N, Moog U, Lorenz-Depiereux B, Eck S, Hempel M, Wagenstaller J, Gawthrope A, Monaco AP et al (2010) Identification of FOXP1 deletions in three unrelated patients with mental retardation and significant speech and language deficits. Hum Mutat 31: E1851-1860

Hsu AL, Murphy CT, Kenyon C (2003) Regulation of aging and age-related disease by DAF-16 and heat-shock factor. Science 300: 1142-1145

Iordanskiy S, Zhao Y, Dubrovsky L, Iordanskaya T, Chen M, Liang D, Bukrinsky M (2004) Heat shock protein 70 protects cells from cell cycle arrest and apoptosis induced by human immunodeficiency virus type 1 viral protein R. J Virol 78: 9697-9704

Jaattela M, Wissing D, Bauer PA, Li GC (1992) Major heat shock protein hsp70 protects tumor cells from tumor necrosis factor cytotoxicity. EMBO J 11: 3507-3512

Jensen TJ, Loo MA, Pind S, Williams DB, Goldberg AL, Riordan JR (1995) Multiple proteolytic systems, including the proteasome, contribute to CFTR processing. Cell 83: 129-135

Kaestner KH, Knochel W, Martinez DE (2000) Unified nomenclature for the winged helix/forkhead transcription factors. Genes Dev 14: 142-146

Katoh M (2013) Functional and cancer genomics of ASXL family members. Br J Cancer 109: 299-306 
Kim D, Paggi JM, Park C, Bennett C, Salzberg SL (2019) Graph-based genome alignment and genotyping with HISAT2 and HISAT-genotype. Nat Biotechnol 37: 907-915

Kim YE, Hipp MS, Bracher A, Hayer-Hartl M, Hartl FU (2013) Molecular chaperone functions in protein folding and proteostasis. Annu Rev Biochem 82: 323-355

Kim YE, Hosp F, Frottin F, Ge H, Mann M, Hayer-Hartl M, Hartl FU (2016) Soluble Oligomers of PolyQ-Expanded Huntingtin Target a Multiplicity of Key Cellular Factors. Mol Cell 63: 951964

Kopito RR (2000) Aggresomes, inclusion bodies and protein aggregation. Trends Cell Biol 10: 524-530

Kops GJ, Dansen TB, Polderman PE, Saarloos I, Wirtz KW, Coffer PJ, Huang TT, Bos JL, Medema RH, Burgering BM (2002) Forkhead transcription factor FOXO3a protects quiescent cells from oxidative stress. Nature 419: 316-321

Kortum F, Das S, Flindt M, Morris-Rosendahl DJ, Stefanova I, Goldstein A, Horn D, Klopocki E, Kluger G, Martin P et al (2011) The core FOXG1 syndrome phenotype consists of postnatal microcephaly, severe mental retardation, absent language, dyskinesia, and corpus callosum hypogenesis. J Med Genet 48: 396-406

Kuwahara T, Tonegawa R, Ito G, Mitani S, Iwatsubo T (2012) Phosphorylation of alpha-synuclein protein at Ser-129 reduces neuronal dysfunction by lowering its membrane binding property in Caenorhabditis elegans. J Biol Chem 287: 7098-7109

Lai CS, Fisher SE, Hurst JA, Levy ER, Hodgson S, Fox M, Jeremiah S, Povey S, Jamison DC, Green ED et al (2000) The SPCH1 region on human 7q31: genomic characterization of the critical interval and localization of translocations associated with speech and language disorder. Am J Hum Genet 67: 357-368

Lehmann OJ, Sowden JC, Carlsson P, Jordan T, Bhattacharya SS (2003) Fox's in development and disease. Trends Genet 19: 339-344

Lein ES, Hawrylycz MJ, Ao N, Ayres M, Bensinger A, Bernard A, Boe AF, Boguski MS, Brockway KS, Byrnes EJ et al (2007) Genome-wide atlas of gene expression in the adult mouse brain. Nature 445: 168-176

Li H, Handsaker B, Wysoker A, Fennell T, Ruan J, Homer N, Marth G, Abecasis G, Durbin R, Genome Project Data Processing S (2009) The Sequence Alignment/Map format and SAMtools. Bioinformatics 25: 2078-2079 
Love MI, Huber W, Anders S (2014) Moderated estimation of fold change and dispersion for RNA-seq data with DESeq2. Genome Biol 15: 550

MacDermot KD, Bonora E, Sykes N, Coupe AM, Lai CS, Vernes SC, Vargha-Khadem F, McKenzie F, Smith RL, Monaco AP et al (2005) Identification of FOXP2 truncation as a novel cause of developmental speech and language deficits. Am J Hum Genet 76: 1074-1080

Markossian KA, Kurganov BI (2004) Protein folding, misfolding, and aggregation. Formation of inclusion bodies and aggresomes. Biochemistry (Mosc) 69: 971-984

Mazet F, Yu JK, Liberles DA, Holland LZ, Shimeld SM (2003) Phylogenetic relationships of the Fox (Forkhead) gene family in the Bilateria. Gene 316: 79-89

McCarroll SA, Hyman SE (2013) Progress in the genetics of polygenic brain disorders: significant new challenges for neurobiology. Neuron 80: 578-587

Morley JF, Morimoto RI (2004) Regulation of longevity in Caenorhabditis elegans by heat shock factor and molecular chaperones. Mol Biol Cell 15: 657-664

Mukamel Z, Konopka G, Wexler E, Osborn GE, Dong H, Bergman MY, Levitt P, Geschwind DH (2011) Regulation of MET by FOXP2, genes implicated in higher cognitive dysfunction and autism risk. J Neurosci 31: 11437-11442

Murphy CT, McCarroll SA, Bargmann CI, Fraser A, Kamath RS, Ahringer J, Li H, Kenyon C (2003) Genes that act downstream of DAF-16 to influence the lifespan of Caenorhabditis elegans. Nature 424: 277-283

Nakagawa S, Gisselbrecht SS, Rogers JM, Hartl DL, Bulyk ML (2013) DNA-binding specificity changes in the evolution of forkhead transcription factors. Proc Natl Acad Sci U S A 110: 1234912354

Nollen EA, Morimoto RI (2002) Chaperoning signaling pathways: molecular chaperones as stresssensing 'heat shock' proteins. J Cell Sci 115: 2809-2816

Nudel R, Newbury DF (2013) Foxp2. Wiley Interdiscip Rev Cogn Sci 4: 547-560

Parenti I, Rabaneda LG, Schoen H, Novarino G (2020) Neurodevelopmental Disorders: From Genetics to Functional Pathways. Trends Neurosci 43: 608-621

Park BJ, Lim YS, Lee HJ, Eum WS, Park J, Han KH, Choi SY, Lee KS (2009) Anti-oxidative effects of Phellinus linteus and red ginseng extracts on oxidative stress-induced DNA damage. BMB Rep 42: 500-505 
Petit FG, Kervarrec C, Jamin SP, Smagulova F, Hao C, Becker E, Jegou B, Chalmel F, Primig M (2015) Combining RNA and protein profiling data with network interactions identifies genes associated with spermatogenesis in mouse and human. Biol Reprod 92: 71

Pommerenke C, Hauer V, Zaborski M, MacLeod RA, Nagel S, Amini RM, Berglund M, Geffers R, Drexler HG, Quentmeier H (2016) Chromosome 11q23 aberrations activating FOXR1 in Bcell lymphoma. Blood Cancer J 6: e433

Pringsheim M, Mitter D, Schroder S, Warthemann R, Plumacher K, Kluger G, Baethmann M, Bast T, Braun S, Buttel HM et al (2019) Structural brain anomalies in patients with FOXG1 syndrome and in Foxg1+/- mice. Ann Clin Transl Neurol 6: 655-668

Raynes R, Pomatto LC, Davies KJ (2016) Degradation of oxidized proteins by the proteasome: Distinguishing between the 20S, 26S, and immunoproteasome proteolytic pathways. Mol Aspects Med 50: 41-55

Sabatini DM, Erdjument-Bromage H, Lui M, Tempst P, Snyder SH (1994) RAFT1: a mammalian protein that binds to FKBP12 in a rapamycin-dependent fashion and is homologous to yeast TORs. Cell 78: 35-43

Santo EE, Ebus ME, Koster J, Schulte JH, Lakeman A, van Sluis P, Vermeulen J, Gisselsson D, Ora I, Lindner S et al (2012) Oncogenic activation of FOXR1 by 11q23 intrachromosomal deletion-fusions in neuroblastoma. Oncogene 31: 1571-1581

Scandalios JG (2005) Oxidative stress: molecular perception and transduction of signals triggering antioxidant gene defenses. Braz J Med Biol Res 38: 995-1014

Scherzinger E, Lurz R, Turmaine M, Mangiarini L, Hollenbach B, Hasenbank R, Bates GP, Davies SW, Lehrach H, Wanker EE (1997) Huntingtin-encoded polyglutamine expansions form amyloid-like protein aggregates in vitro and in vivo. Cell 90: 549-558

Seltzer LE, Paciorkowski AR (2014) Genetic disorders associated with postnatal microcephaly. Am J Med Genet C Semin Med Genet 166C: 140-155

Shen D, Coleman J, Chan E, Nicholson TP, Dai L, Sheppard PW, Patton WF (2011) Novel celland tissue-based assays for detecting misfolded and aggregated protein accumulation within aggresomes and inclusion bodies. Cell Biochem Biophys 60: 173-185

Shoichet SA, Kunde SA, Viertel P, Schell-Apacik C, von Voss H, Tommerup N, Ropers HH, Kalscheuer VM (2005) Haploinsufficiency of novel FOXG1B variants in a patient with severe mental retardation, brain malformations and microcephaly. Hum Genet 117: 536-544 
Singh V, Aballay A (2009) Regulation of DAF-16-mediated Innate Immunity in Caenorhabditis elegans. J Biol Chem 284: 35580-35587

Stenoien DL, Mielke M, Mancini MA (2002) Intranuclear ataxin1 inclusions contain both fastand slow-exchanging components. Nat Cell Biol 4: 806-810

Subramanian A, Tamayo P, Mootha VK, Mukherjee S, Ebert BL, Gillette MA, Paulovich A, Pomeroy SL, Golub TR, Lander ES et al (2005) Gene set enrichment analysis: a knowledgebased approach for interpreting genome-wide expression profiles. Proc Natl Acad Sci U S A 102: $15545-15550$

Takahashi H, Takahashi K, Liu FC (2009) FOXP genes, neural development, speech and language disorders. Adv Exp Med Biol 665: 117-129

Tarlungeanu DC, Novarino G (2018) Genomics in neurodevelopmental disorders: an avenue to personalized medicine. Exp Mol Med 50: 100

Teer JK, Bonnycastle LL, Chines PS, Hansen NF, Aoyama N, Swift AJ, Abaan HO, Albert TJ, Program NCS, Margulies EH et al (2010) Systematic comparison of three genomic enrichment methods for massively parallel DNA sequencing. Genome Res 20: 1420-1431

van Dongen MJ, Cederberg A, Carlsson P, Enerback S, Wikstrom M (2000) Solution structure and dynamics of the DNA-binding domain of the adipocyte-transcription factor FREAC-11. J Mol Biol 296: 351-359

Voellmy R (1994) Transduction of the stress signal and mechanisms of transcriptional regulation of heat shock/stress protein gene expression in higher eukaryotes. Crit Rev Eukaryot Gene Expr 4: $357-401$

Ward CL, Omura S, Kopito RR (1995) Degradation of CFTR by the ubiquitin-proteasome pathway. Cell 83: 121-127

Weigel D, Jurgens G, Kuttner F, Seifert E, Jackle H (1989) The homeotic gene fork head encodes a nuclear protein and is expressed in the terminal regions of the Drosophila embryo. Cell 57: $645-658$

Xuan S, Baptista CA, Balas G, Tao W, Soares VC, Lai E (1995) Winged helix transcription factor BF-1 is essential for the development of the cerebral hemispheres. Neuron 14: 1141-1152

Yao J, Liu Y, Lo R, Tretjakoff I, Peterson A, Stifani S (2000) Disrupted development of the cerebral hemispheres in transgenic mice expressing the mammalian Groucho homologue transducin-like-enhancer of split 1 in postmitotic neurons. Mech Dev 93: 105-115 
Yuan H, Hansen KB, Zhang J, Pierson TM, Markello TC, Fajardo KV, Holloman CM, Golas G, Adams DR, Boerkoel CF et al (2014) Functional analysis of a de novo GRIN2A missense mutation associated with early-onset epileptic encephalopathy. Nat Commun 5: 3251 


\section{Figure legends}

Figure 1. Neuroimaging and identification of a de novo FOXR1 missense variant in a proband with microcephaly and brain atrophy

A. Top images, MRI of mid-sagittal images of the proband at 1 and 5 years old showing hypoplasia of the pons (arrows) and cerebellum. Bottom images, Horizontal view of the proband at 1 and 5 years old showing dilation of ventricle indicated by the arrows.

B. Pedigree of the family where the letter P in red (black square) indicates the proband.

C. Sanger sequence analysis confirming the de novo FOXR1 variant. Sequence chromatograms demonstrate the presence of the heterozygous variant in the proband, II-4 (indicated by the red arrow) and the reference allele in both parents and siblings (green arrows). Letters on top indicate amino acid residues $(\mathrm{Q}=$ glutamine, $\mathrm{C}=$ cysteine, $\mathrm{M}=$ methionine, $\mathrm{L}=$ leucine, $\mathrm{S}=$ serine, $\mathrm{P}=$ proline).

\section{Figure 2. The M280L mutant destabilizes FOXR1 protein}

A. Representative immunoblots and quantitative analysis of FOXR1 from HEK293T cells transfected with pCMV-SPORT6 human FOXR1 WT or M280L mutant. GAPDH served as a loading control. Graph represents FOXR1 over GAPDH normalized to WT. Unpaired t-test (n $=6$ independent experiments, $* * * \mathrm{p}<0.0001)$.

B. Representative immunoblot and quantitative analysis of FOXR1 from HEK293T cells transfected with GFP, GFP-tagged human FOXR1 WT or M280L mutant. GAPDH served as a loading control. Graph represents FOXR1 over GAPDH normalized to WT. Unpaired t-test (n $=5$ independent experiments, $* * * \mathrm{p}<0.0001)$.

C. Representative immunoblot and quantitative analysis of FOXR1 from COS7 cells transfected with GFP, GFP-tagged human FOXR1 WT or M280L mutant. GAPDH served as a loading control. Graph represents FOXR1 over GAPDH normalized to WT. Unpaired $t$-test $(\mathrm{n}=4$ independent experiments, $* * \mathrm{p}=0.0013)$.

D. Representative immunoblots and quantitative analysis of cytoplasmic (c) and nuclear (n) fractions of FOXR1 from HEK293T cells transfected with pCMV-SPORT6 or GFP-tagged human FOXR1 WT and M280L. GAPDH and Histone H3 served as cytoplasmic and nuclear loading markers, respectively. Graph represents FOXR1 over GAPDH normalized to WT. Unpaired $t$-test $(\mathrm{n}=5$ independent experiments, $* * * \mathrm{p}<0.0001)$. The percentages of total cellular FOXR1 is the cytoplasmic and nuclear fractions were determined. 
E. Quantitative PCR (qPCR) to quantify FOXR1 mRNA levels from HEK293T cells transfected with GFP, GFP-tagged human FOXR1 WT or M280L mutant. Graph represents relative FOXR1 mRNA expression normalized to GFP.

F. Representative immunoblot and quantitative analysis of FOXR1 from HEK293T cells transfected with GFP-tagged human FOXR1 WT or M280L mutant. Protein stability was monitored by quantitative immunoblotting after blocking with proteasome inhibitor MG132. Graph represents FOXR1 over GAPDH normalized to untreated WT. One-way ANOVA ( $\mathrm{n}=$ 4 independent experiments, $* \mathrm{p}=0.0143, * * \mathrm{p}=0.0012, * * * \mathrm{p}=0.0003)$.

G. Representative immunoblot and quantitative analysis of FOXR1 from HEK293T cells transfected with GFP-tagged human FOXR1 WT, M280L mutant or FOXR1 C-terminal truncation mutant lacking the last 12 amino acids ( $\Delta 280-292)$. Protein stability was monitored for FOXR1 $\triangle 280-292$ mutant by blocking with MG132. GAPDH served as a loading control. Graph represents FOXR1 over GAPDH normalized to untreated WT. One-way ANOVA $(\mathrm{n}=$ 3 independent experiments, $* * * \mathrm{p}<0.0001)$.

\section{Figure 3. The M280L variant induced nuclear puncta phenotype}

A. Fluorescence images of HEK293T cells transfected with GFP or GFP-tagged human FOXR1 WT, M280L or $\Delta 280-292$ mutants. DAPI (blue) served as a nuclear marker. Scale bar $=20 \mu \mathrm{m}$.

B. Fluorescence images of HEK293T cells transfected with GFP-tagged M280L mutant showing a range of nuclear puncta phenotypes. Scale bar $=5 \mu \mathrm{m}$.

C. Quantitative analysis on the percentage of cells expressing FOXR1 puncta phenotype. One-way ANOVA ( $\mathrm{n}=3$ independent experiments, $* * \mathrm{p}=0.0048, * * * \mathrm{p}=0.0002)$.

D. Correlation analysis of the average size of the aggregate to the number of puncta per nucleus.

\section{Figure 4. RNAseq analysis of FOXR1 wild-type and M280L mutant}

A. Heatmap of hierarchical clustering indicates differentially-expressed genes (rows) between GFP, GFP-tagged FOXR1 WT and M280L (fold-change $>2, \mathrm{p}<0.05$ ). Red indicates upregulated genes and blue indicates down-regulated genes.

B. Heatmap of gene cluster ' $E$ ' indicates differentially-expressed genes (rows) that are upregulated in FOXR1 WT and down-regulated in M280L.

C. Distribution of gene ontology (GO) terms annotated in biological processes of highly-regulated genes in FOXR1 WT and down-regulated in M280L. 
D. Volcano plot of differentially expressed genes that were up-regulated between FOXR1 WT versus GFP control. Significantly up-regulated genes are in red while down-regulated genes are in blue. Non-significant genes are in gray.

E. Volcano plot of differentially expressed genes that were down-regulated between FOXR1 M280L versus WT. Significantly down-regulated genes are in blue and up-regulated are in red. Non-significant genes are in gray.

F. Heatmap of gene cluster ' $E$ ' highlighting several chaperone proteins that were differentially expressed in FOXR1 WT and down-regulated in M280L.

G. Quantitative real-time PCR verifying the RNAseq analysis showing FOXR1 drives expression of HSPA6, HSPA1A and DHRS2 and are misregulated in the M280L mutant. Graph represents relative expression normalized to GFP. One-way ANOVA $\left(\mathrm{n}=3\right.$ independent experiments, ${ }^{*} \mathrm{p}$ $<0.05, * * \mathrm{p}<0.005, * * * \mathrm{p}<0.0001)$.

\section{Figure 5. Human DNA binding-site motifs bound by FOXR1}

A. FOXR1 response elements showing consensus primary and secondary sequences bound by FOXR1 (adapted from Nakagawa et al., 2013).

B. Putative FOXR1 response elements are denoted in the promoters of three of the top-regulated FOXR1-targeted genes: HSPA6, HSPA1A and DHRS2.

C. Dual luciferase reporter assays where GFP control, FOXR1 WT or M280L were co-transfected into HEK293T cells with the corresponding HSPA6, HSPA1A and DHRS2 luciferase reporters. Data are plotted as luciferase activity normalized to GFP control. One-way ANOVA ( $n=3$ independent experiments, $* \mathrm{p}<0.05, * * \mathrm{p}<0.005, * * * \mathrm{p}<0.0001)$.

D. Consensus primary sequences bound by HSF1. The putative HSF1 response elements are denoted in the promoter of FOXR1. Dual luciferase reporter assays in HEK293T cells comparing FOXR1 promoter activation to a FOXR1 mutant where two residues of the HSF1 response elements in FOXR1 were mutated from TT to GG (mutant). Data was plotted as luciferase activity normalized to GFP control. One-way ANOVA ( $\mathrm{n}=3$ independent experiments, $* * \mathrm{p}=0.0062$ ).

\section{Figure 6. FOXR1 expression is increased in response to cellular stress}

A. Representative immunoblots and quantitative analysis for FOXR1 from HEK293T cells transfected with GFP, GFP-tagged FOXR1 WT or M280L mutant in response to serum and 
$\mathrm{CO}_{2}$ deprivation. GAPDH served as loading control. Graph represents FOXR1 over GAPDH normalized to untreated WT. One-way ANOVA $\left(n=4\right.$ independent experiments, $\left.{ }^{*} * \mathrm{p}<0.005\right)$.

B. Fluorescence images of HEK293T cells transfected with GFP-tagged human FOXR1 WT or $\mathrm{M} 280 \mathrm{~L}$ in response to serum and $\mathrm{CO}_{2}$ deprivation. Scale bar $=20 \mu \mathrm{m}$.

C. Fluorescence images of HEK293T cells transfected with GFP-tagged human FOXR1 WT or M280L and treated with PMA, a NADPH oxidase activator known to enhance reactive oxygen species (ROS). Cells were fixed after 24 hours of treatment and assessed for ROS generation using CellROX, a photostable ROS sensor. Scale bar $=20 \mu \mathrm{m}$.

D. Representative immunoblots and quantitative analysis of HEK293T cells following PMA treatment showing an increase in FOXR1 expression. Graph represents FOXR1 over GAPDH normalized to untreated WT. One-way ANOVA $(n=5$ independent experiments, *** $\mathrm{p}<0.0005)$.

E. Quantitative analysis of HSPA6, HSPA1A and DHRS2 protein levels from HEK293T cells transfected with GFP, GFP-tagged human FOXR1 WT or M280L. Graph represents protein of interest over GAPDH normalized to GFP. One-way ANOVA ( $n=4-6$ independent experiments, $* \mathrm{p}<0.05, * * \mathrm{p}<0.005)$.

F. Quantitative analysis of HSPA6, HSPA1A and DHRS2 protein levels from HEK293T cells transfected with GFP, GFP-tagged human FOXR1 WT or M280L and treated with PMA. Graph represents protein of interest over GAPDH normalized to GFP. One-way ANOVA ( $\mathrm{n}=$ 2-3 independent experiments, $* \mathrm{p}<0.05)$.

\section{Figure 7. M280L nuclear aggregates are insoluble misfolded proteins}

A. Fluorescence images of HEK293T cells transfected with GFP-tagged human FOXR1 WT or M280L and treated with PMA. Cells were fixed after 24 hours of treatment and immunolabel with Proteostat marker. White square box in the middle panels indicate images presented in the bottom panel at higher magnification. Top and middle panels, scale bar $=20 \mu \mathrm{m}$. Bottom panels, scale bar $=10 \mu \mathrm{m}$.

B. Fluorescence images of HEK293T cells transfected with GFP-tagged M280L and treated with MG132. Cells were fixed after 24 hours of treatment and immunolabel with Proteostat marker. Scale bar $=10 \mu \mathrm{m}$. 
C. Time-lapse imaging of HEK293T cells transfected with GFP-tagged M280L. Top panel represent images showing nuclear aggregates undergoing extensive movements and fusions. Bottom panel illustrate schematic drawings of the fusion events. Scale bar $=5 \mu \mathrm{m}$.

D. FOXR1 was sequentially extracted with Tris-HCl, Triton X-100, Sarkosyl and SDS in this order. Quantification shows that the amount of FOXR1 in the SDS fraction was significantly higher in the M280L mutant when compared to the overall Tris-HCl total fraction. One-way ANOVA ( $\mathrm{n}=2$ independent experiments, $* * \mathrm{p}<0.005, * * * \mathrm{p}<0.0001)$. 


\section{Appendix figure legends}

Appendix Table 1. Summary of clinical features of the proband with a de novo variant in FOXR1.

Appendix Figure 1. Alignment of cross-species FOXR1 sequences showing the conserved methionine residue (indicated in red) within a highly conserved region of amino acid sequence.

Appendix Figure 2. Fluorescence images of COS7 cells transfected with GFP or GFP-tagged plasmids of human FOXR1 WT or M280L. DAPI (blue) served as a nuclear marker. Scale bar = $20 \mu \mathrm{m}$.

Appendix Figure 3. RNAseq analysis

A. PCA plot of the three groups clustered separately in multidimensional scaling analyses. Groups of samples analyzed using Principal Component Analysis (PCA) plots where replicates are clustered together and clusters from different conditions are separated.

B. B, Pie chart showing the distribution of 2644 differentially-expressed genes between GFP and FOXR1 WT and 735 differentially-expressed genes between FOXR1 WT and M280L.

Appendix Figure 4. Gene ontology (GO) enrichment analysis between FOXR1 WT and M280L and GFP control and FOXR1 WT. Normalized enrichment scores indicate the distribution of biological processes across a list of genes ranked by hypergeometrical score. Higher enrichment scores indicate a shift of genes belonging to certain GO categories towards either end of the ranked list, representing up or down-regulation (positive or negative values, respectively).

Appendix Figure 5. Promoter sequences showing FOXR1 response elements lie upstream of the HSEs in both the HSPA6 and HSPA1A promoters. In addition, we identified a consensus sequence for binding by HSF1 within the promoter region of FOXR1.

Appendix Movie 1. Movie of M280L in response to $\mathrm{CO}_{2}$ stress.

Appendix Movie 2. Movie of M280L in response to PMA treatment.

Appendix Movie 3. Movie of M280L in response to PMA treatment at high magnification. 
Figure 1

\section{A}
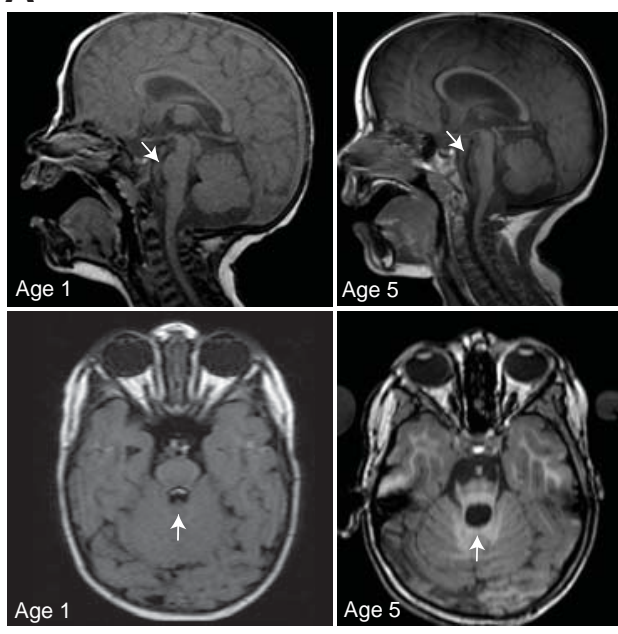

B

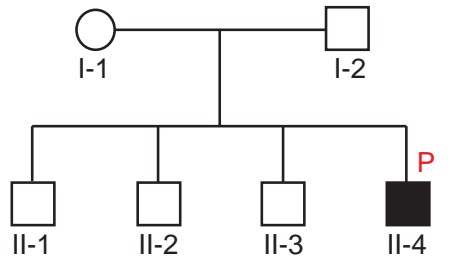

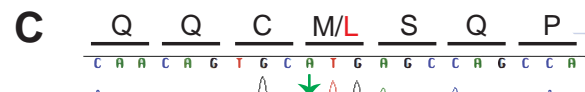
$\stackrel{\rightarrow}{-}$
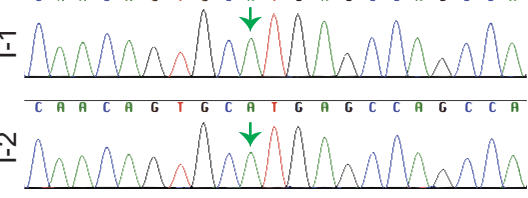

$\stackrel{+}{=}$
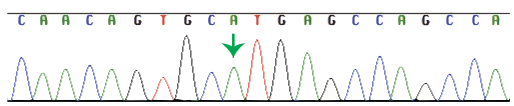

$\stackrel{\sim}{\stackrel{1}{=}}$

C A A C A G I G C A Y G A G C C A G C C A

$\stackrel{m}{=}$

C A A C A G I G C A I G A C C C A G C C A

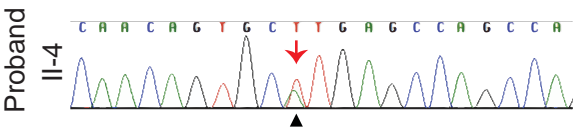


Figure 2
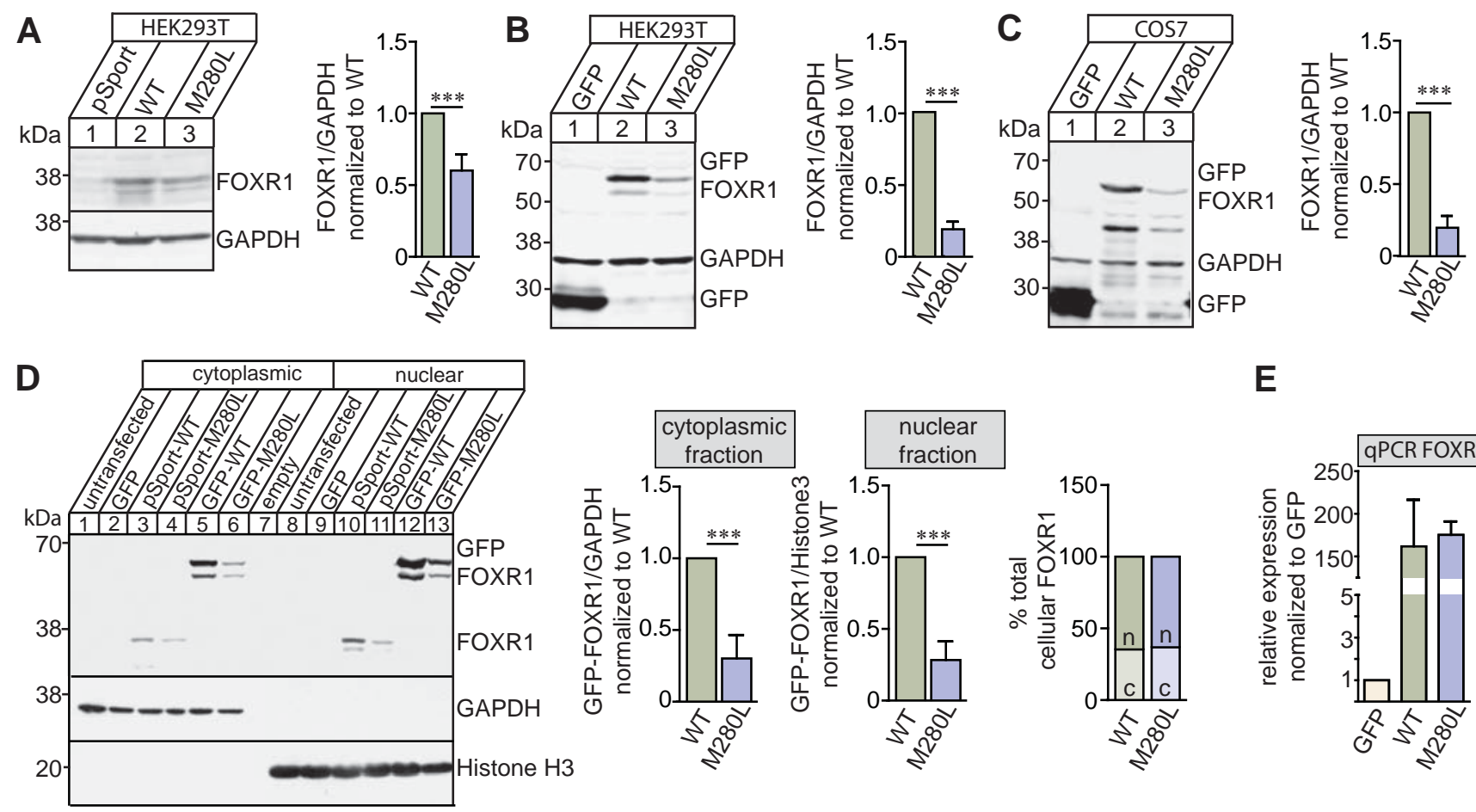

E
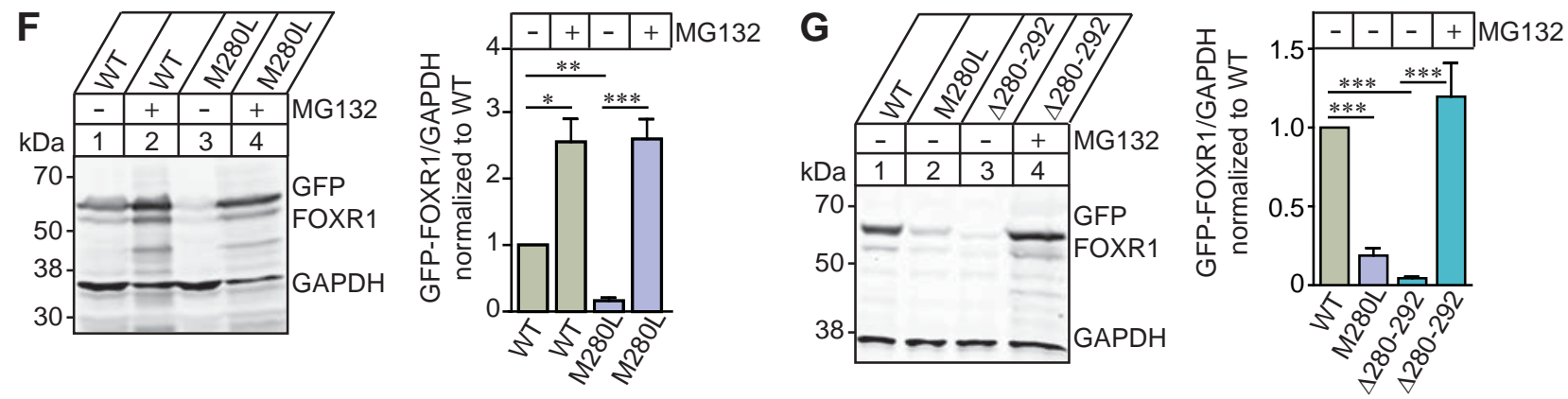
Figure 3

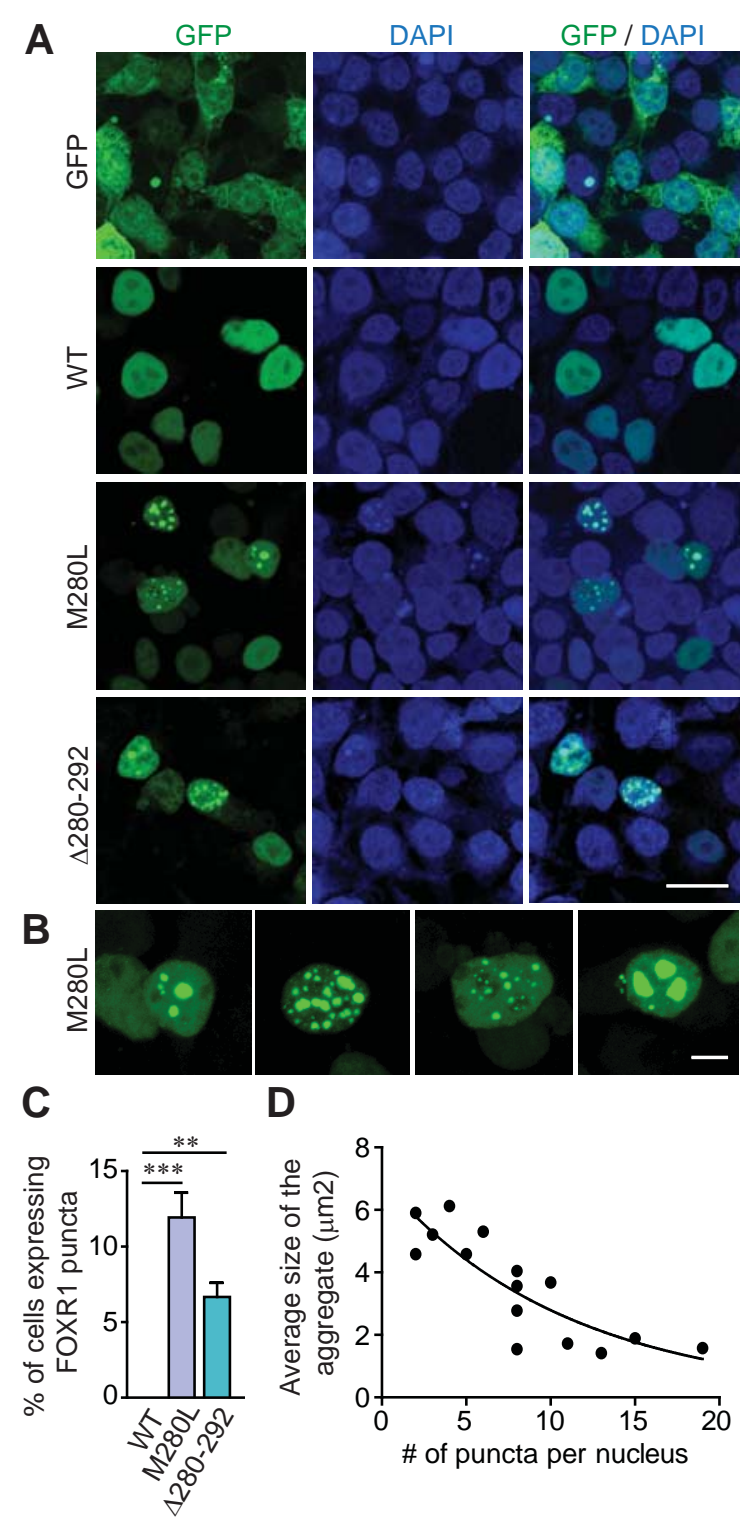


bioRxiv preprint doi: https://doi.org/10.1101/2020.11.03.366740; this version posted November 3, 2020. The copyright holder for this preprint (which was not certified by peer review) is the author/funder. All rights reserved. No reuse allowed without permission.

Figure 4

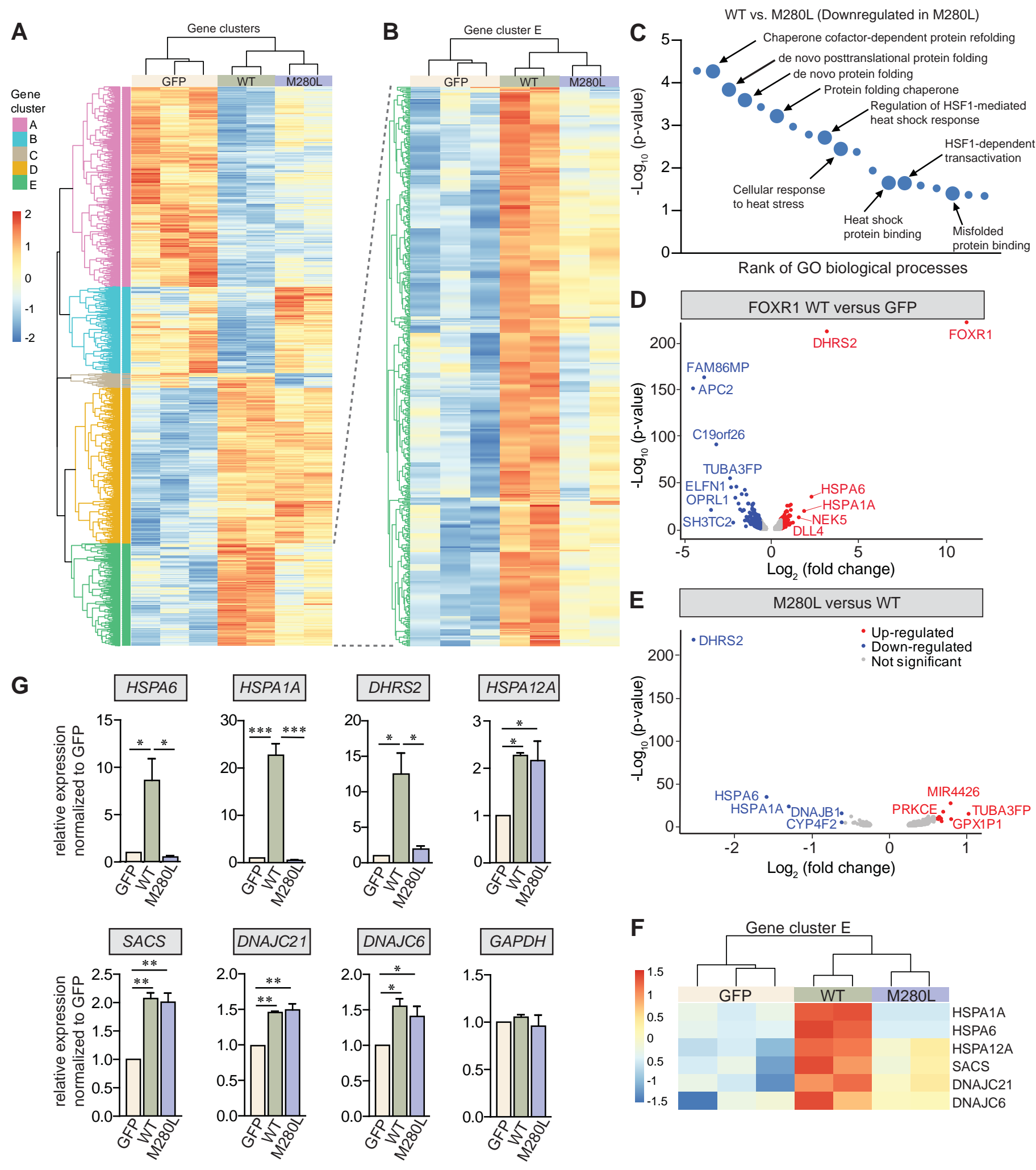


Figure 5

A FOXR1 response elements

Primary Motif Secondary Motif

A

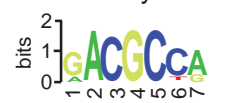

B H. sap HSPA6

$5^{\prime}$-ctcggggataCAAACAgtggggagaa-3'

$-1097$

$-1069$

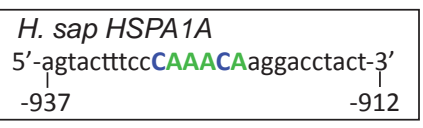

\begin{tabular}{|c|c|}
\hline $\begin{array}{l}\text { H. sa } \\
5^{\prime} \text {-gct }\end{array}$ & tcag-3' \\
\hline-2630 & -2606 \\
\hline $\begin{array}{l}5 \text { '-gttc } \\
-2487\end{array}$ & \\
\hline
\end{tabular}

C

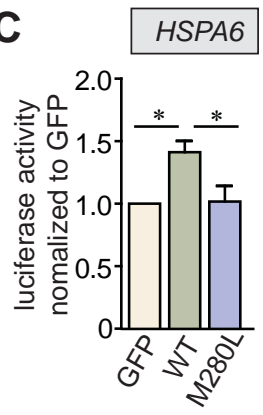

D HSF1 (Motif Regressor on GSE7665)
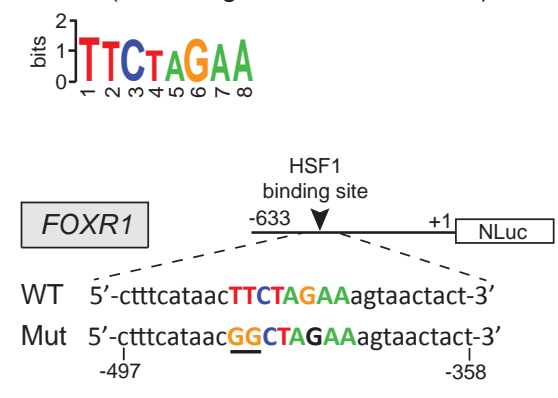

HSPA1A

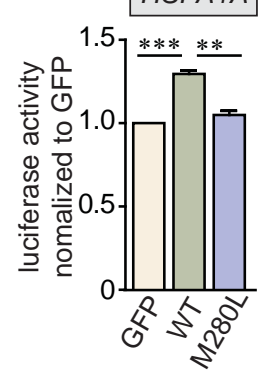

65)
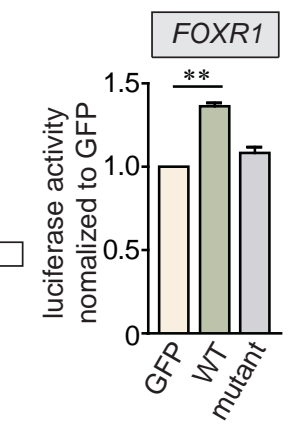
Figure 6
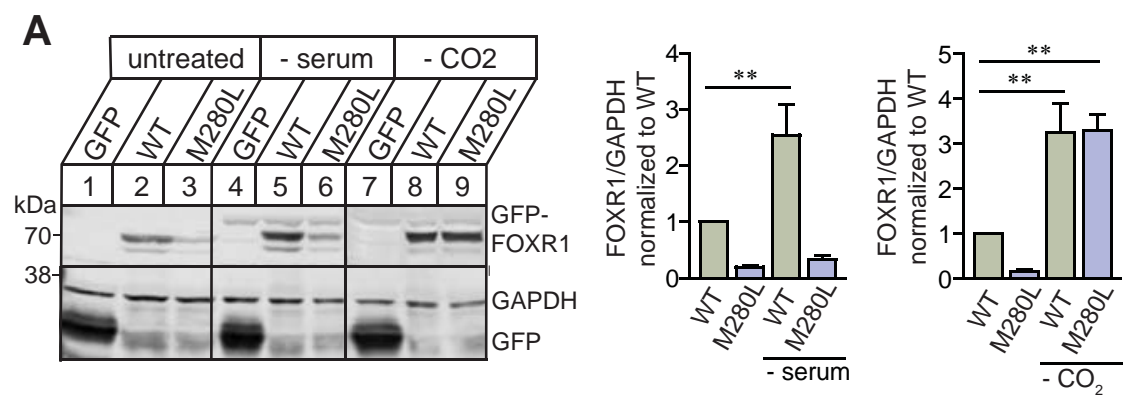
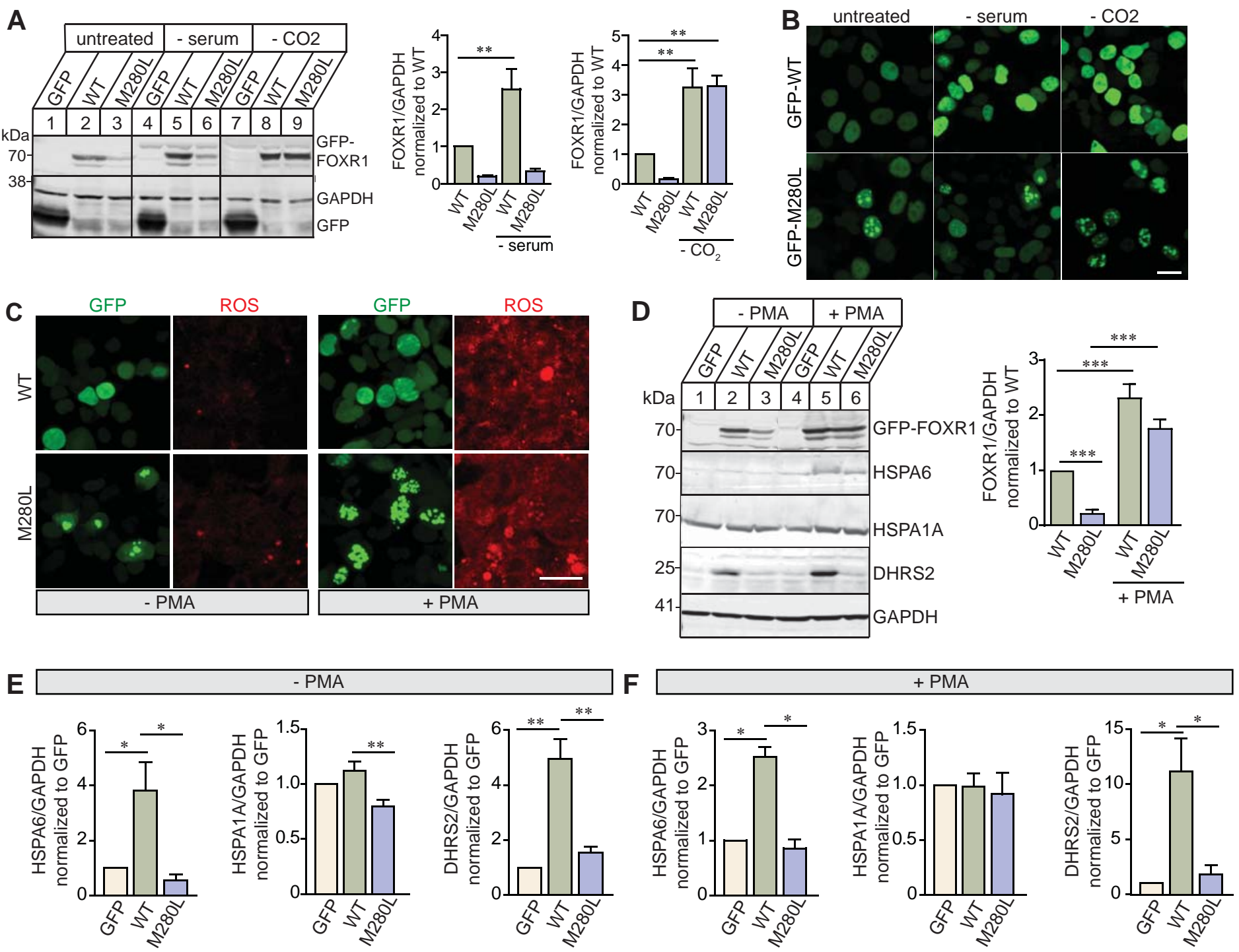
Figure 7

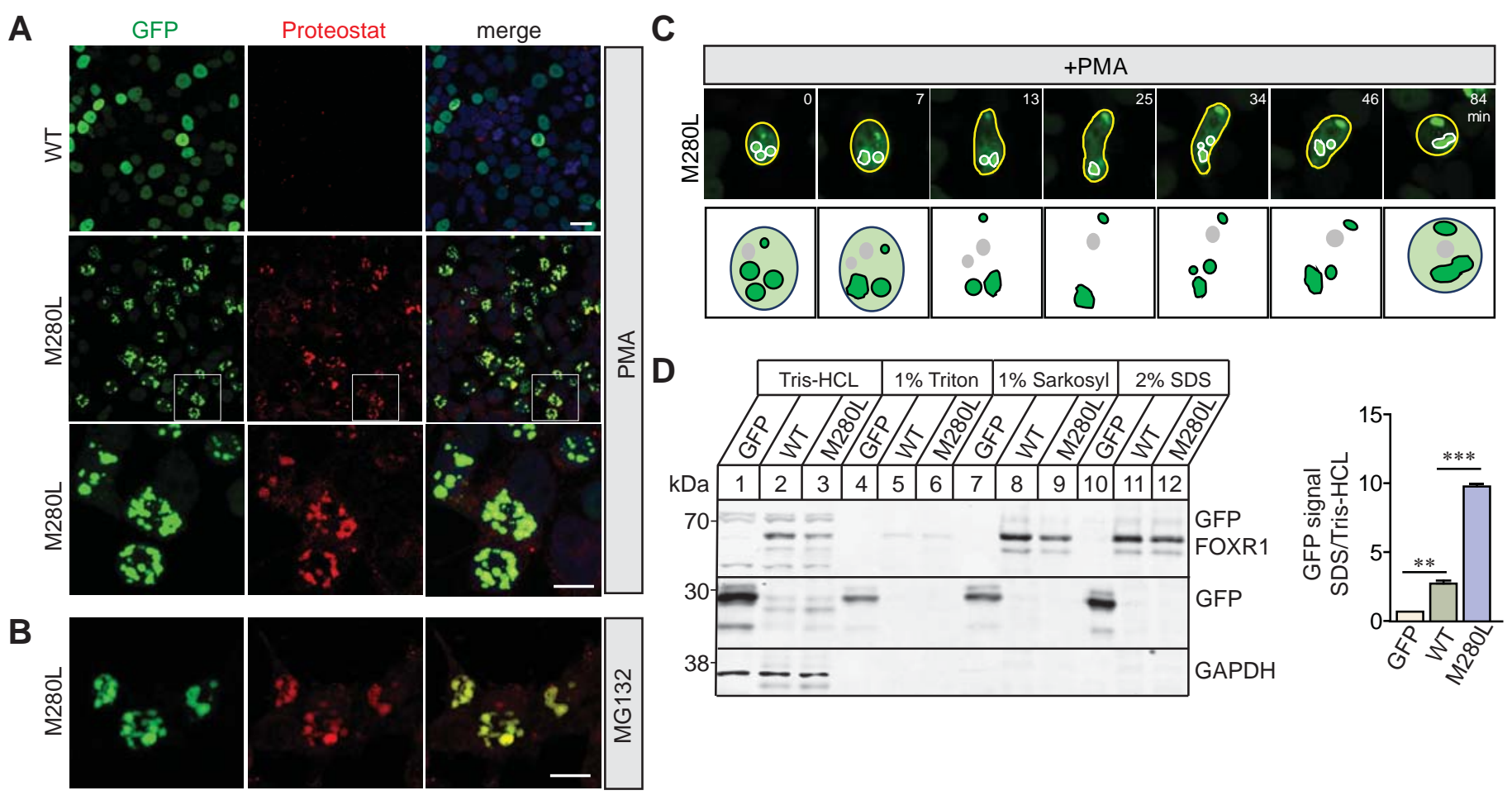

